\title{
Triple Hilbert transforms along polynomial surfaces in $\mathbb{R}^{4}$
}

Anthony Carbery, Stephen Wainger and James Wright

\begin{abstract}
We investigate the $L^{2}$ boundedness of the triple Hilbert transform along the surface given by the graph of a real polynomial $P$ of three variables. We are interested in understanding the relationship between the geometric properties of the Newton polyhedron of $P$ and the analytic property of $L^{2}$ boundedness.
\end{abstract}

\section{Introduction}

Let $P(s, t, u)$ be a real-valued polynomial of three real variables. Set $\Gamma(s, t, u)$ $=(s, t, u, P(s, t, u))$. For $x \in \mathbb{R}^{4}$ and $f$ a Schwartz function on $\mathbb{R}^{4}$ set

$$
H f(x)=\lim _{\epsilon \rightarrow 0} \iiint_{\substack{\epsilon \leq|s| \leq 1 \\ \epsilon \leq|t| \leq 1 \\ \epsilon \leq|u| \leq 1}} f(x-\Gamma(s, t, u)) \frac{d s d t d u}{s t u} .
$$

We are interested in conditions on $P$ so that we have the estimate

$$
\|H f\|_{L^{2}\left(\mathbb{R}^{4}\right)} \leq C\|f\|_{L^{2}\left(\mathbb{R}^{4}\right)} .
$$

This problem is motivated and influenced by earlier work of [2] and [4]. Our analysis of the problem relies upon ideas and techniques developed in these papers.

Previously, we had studied the corresponding operator in one lower dimension. That is for $P(s, t)$ a polynomial of two variables, $\Gamma(s, t)=$ $(s, t, P(s, t)), f$ a Schwartz function on $\mathbb{R}^{3}$ and $x$ in $\mathbb{R}^{3}$, we considered

$$
\mathcal{H} f(x)=\lim _{\epsilon \rightarrow 0} \iint_{\substack{\epsilon \leq|s| \leq 1 \\ \epsilon \leq|t| \leq 1}} f(x-\Gamma(s, t)) \frac{d s d t}{s t} .
$$

2000 Mathematics Subject Classification: 42B15.

Keywords: Hilbert transform, Newton polyhedron and oscillatory integrals. 
(Here and in what follows, the notation (Roman) $H$ will always refer to operators on $\mathbb{R}^{4}$ while (calligraphic) $\mathcal{H}$ will refer to operators on $\mathbb{R}^{3}$.)

The boundedness of $\mathcal{H}$ (and in fact in any $L^{p}, 1<p<\infty$ ) is determined by the Newton polygon of $P$. The Newton polygon of $P$ is obtained in the following way. We plot the points $(a, b)$ for each $(a, b)$ such that the monomial $s^{a} t^{b}$ occurs in $P$ with a non-zero coefficient. For such an $(a, b)$ let $R_{a, b}=\left\{x, y \in \mathbb{R}^{2}: x \geq a, y \geq b\right\}$. Then the Newton polygon of $P$ is the smallest convex set containing $\bigcup R_{a, b}$. The Newton polygon of $P$ is an (unbounded) polygon. In [1] we obtained the following result:

Theorem A. Assume $P(0,0)=\nabla P(0,0)=0$. Then for any $p, 1<p<\infty$,

$$
\|\mathcal{H} f\|_{L^{p}\left(\mathbb{R}^{3}\right)} \leq C(p)\|f\|_{L^{p}\left(\mathbb{R}^{3}\right)}
$$

if and only if for each vertex $(a, b)$ of the Newton polyhedron of $P$ at least one of $a$ and $b$ is even.

Theorem A suggests that the boundedness of $H$ should depend only on the Newton polyhedron of $P$. For $P$ a polynomial of three variables we form the Newton polyhedron of $P$ in the following manner. For each monomial $s^{a} t^{b} u^{d}$ which occurs in $P$ with a non-zero coefficient, we set

$$
R_{(a, b, d)}=\left\{(x, y, z) \in \mathbb{R}^{3}: x \geq a, y \geq b, z \geq d\right\} .
$$

Then the Newton polyhedron of $P$ is the smallest convex set containing the union of the $R_{(a, b, d)}$. In fact, it transpires that the boundedness of $H$ is not determined by the Newton polyhedron alone; indeed it may depend upon the actual coefficients of the polynomial in question. This means that the the condition for boundedness of $H$ has a much more complicated nature than in the setting of Theorem A.

In order to understand the situation in $\mathbb{R}^{4}$, it is convenient to review the strategy of the proof of Theorem A. For simplicity assume that $P(s, 0)=$ $P(0, t)=0$. For each vertex $(a, b)$ of the Newton polyhedron of $P$ we consider the corresponding monomial $V=e s^{a} t^{b}$ and we set

$$
\mathcal{H}_{\mathcal{R}}^{V} f\left(x_{1}, x_{2}, x_{3}\right)=\int_{\mathcal{R}} f\left(x_{1}-s, x_{2}-t, x_{3}-e s^{a} t^{b}\right) \frac{d s d t}{s t} .
$$

Here $\mathcal{R}=\left\{(s, t):|s|^{a}|t|^{b} \geq|s|^{\alpha}|t|^{\beta}\right.$ for all exponents $(\alpha, \beta)$ arising in $\left.P\right\}$. We then reduce the proof of the boundedness of $\mathcal{H}$ to the boundedness of each $\mathcal{H}_{\mathcal{R}}^{V}$. The boundedness of each $\mathcal{H}_{\mathcal{R}}^{V}$ now follows from Theorem 5.1 of [4]. It turns out that the condition for the boundedness of $\mathcal{H}_{\mathcal{R}}^{V}$ - namely at least one of $a$ and $b$ be even - is the same as that for $\mathcal{H}^{V}$ where

$$
\mathcal{H}^{V} f\left(x_{1}, x_{2}, x_{3}\right)=\int_{\mathbb{R}^{2}} f\left(x_{1}-s, x_{2}-t, x_{3}-e s^{a} t^{b}\right) \frac{d s d t}{s t},
$$


The condition that at least one of $a$ and $b$ be even is equivalent to condition (i) in Theorem 5.1 of [4], and this theorem then ensures the boundedness of $\mathcal{H}_{R}^{V}$.

In four dimensions, we reduce matters to the study of certain "edge operators". Suppose $Q=\sum e_{j} s^{a_{j}} t^{b_{j}} u^{d_{j}}$, where the sum is over all $\left(a_{j}, b_{j}, d_{j}\right)$ such that $e_{j} s^{a_{j}} t^{b_{j}} u^{d_{j}}$ is a monomial of $P$ with $\left(a_{j}, b_{j}, d_{j}\right)$ lying on an edge of the Newton polyhedron of $P$. Then we reduce the proof of the boundedness of $H$ to the boundedness of operators

$$
H_{\mathcal{R}}^{Q} f(x)=\int_{\mathcal{R}} f\left(x-\Gamma_{Q}(s, t, u)\right) \frac{d s d t d u}{s t u}
$$

where $\Gamma_{Q}(s, t, u)=(s, t, u, Q(s, t, u))$, and $\mathcal{R}$ is a region that depends on $Q$ and $P$.

To get some understanding of why Theorem 5.1 of [4] applies to the operators $\mathcal{H}_{\mathcal{R}}^{V}$ in $\mathbb{R}^{3}$ and not to $H_{\mathcal{R}}^{Q}$ in $\mathbb{R}^{4}$, consider

$$
H^{Q} f(x)=\int_{\mathbb{R}^{3}} f\left(x-\Gamma_{Q}(s, t, u)\right) \frac{d s d t d u}{s t u} .
$$

Both this operator and $\mathcal{H}^{V}$ are homogeneous with respect to a two parameter dilation group (assuming $Q$ has at least two monomials). In the case of $\mathcal{H}^{V}$ the dilation group is $D_{\lambda, \eta}$, where

$$
D_{\lambda, \eta}(x, y, z)=\left(\lambda x, \eta y, \lambda^{a} \eta^{b} z\right) .
$$

If the $d_{j}$ are distinct, the dilation group for $H^{Q}$ is

$$
D_{\lambda, \eta}(x, y, z, w)=\left(\lambda x, \eta y, \lambda^{A_{1}} \eta^{A_{2}} z, \lambda^{B_{1}} \eta^{B_{2}} w\right)
$$

where

$$
\begin{aligned}
A_{1} & =\frac{a_{2}-a_{1}}{d_{1}-d_{2}}, & A_{2} & =\frac{b_{2}-b_{1}}{d_{1}-d_{2}} \\
B_{1} & =\frac{a_{2} d_{1}-a_{1} d_{2}}{d_{1}-d_{2}}, & B_{2} & =\frac{b_{2} d_{1}-b_{1} d_{2}}{d_{1}-d_{2}} .
\end{aligned}
$$

In the case of $\mathcal{H}^{V}$ almost every point in $\mathbb{R}^{2}$ is a dilate of a point $(x, y, z)$ such that $x= \pm 1, y^{2}+z^{2}=1$, the set of which we denote by $\bar{\Sigma}$. Then the operator $\mathcal{H}^{V}$ can be formed by integrating dilates of the distribution $\delta\left(z-x^{a} y^{b}\right) \frac{1}{x y}$ (where $\delta$ is the Dirac delta) restricted to $\bar{\Sigma}$. Notice that this distribution restricted to $\bar{\Sigma}$ is not singular and in fact defines a finite measure. Now let $\Sigma=\left\{(x, y, z, w) \in \mathbb{R}^{4}: x= \pm 1\right.$ and $\left.y^{2}+z^{2}+w^{2}=1\right\}$. If $A_{1}, A_{2}, B_{1}$ and $B_{2}$ are all positive then almost every point in $\mathbb{R}^{4}$ is equivalent, 
under the appropriate group of dilations, to a point on $\Sigma$. Unlike the three dimensional case discussed above observe that the distribution

$$
\delta(w-Q(x, y, z)) \frac{1}{x y z}
$$

when restricted to $\Sigma$, does not define a finite measure.

Nevertheless, the Ricci-Stein paper is very suggestive for us in two ways. First to each dilation group, Ricci and Stein associate a matrix $\Lambda$. In the case at hand this is

$$
\Lambda=\left(\begin{array}{cc}
1 & 0 \\
0 & 1 \\
A_{1} & A_{2} \\
B_{1} & B_{2}
\end{array}\right)
$$

The most nondegenerate situation is when each $2 \times 2$ subdeterminant is not zero. This means that when we consider $\left(a_{j}, b_{j}, d_{j}\right)$ corresponding to an edge (assuming the $d_{j}$ are distinct as above), the $a_{j}$ are distinct, the $b_{j}$ are distinct and the projections of the line through $\left(a_{j}, b_{j}, d_{j}\right)$ onto the $x-z$ plane and the $y-z$ plane do not pass through the origin. We shall make these assumptions to put ourselves in this nondegenerate situation and shall also assume that all $a_{j}, b_{j}$ and $d_{j}$ are positive in order to avoid the need for some subsidiary arguments. ${ }^{1}$

Thus we are led to the following assumptions. If $\left(a_{j}, b_{j}, d_{j}\right)$ lie on an edge corresponding to $Q$,

(i) Each $a_{j}, b_{j}$ and $d_{j}$ is positive

(ii) Each sequence $\left\{a_{j}\right\},\left\{b_{j}\right\}$ and $\left\{d_{j}\right\}$ is strictly monotone and

(iii) The projection of the line through the points $\left(a_{j}, b_{j}, d_{j}\right)$ onto a coordinate plane does not pass through the origin.

We shall always assume conditions $(i),(i i)$ and $(i i i)$ for each $Q$ arising from an edge of the Newton polyhedron of $P$.

The second point that we should learn from the Ricci-Stein paper is that the appropriate cancellation condition for the boundedness of $H^{Q}$ should be expressed in terms of the vanishing of an oscillatory integral involving $Q$, and this suggests that the boundedness of $H^{Q}$ may depend on the coefficients of $Q$ and not just the powers $\left(a_{j}, b_{j}, d_{j}\right)$.

Even under these natural assumptions, the situation is quite intricate, and in order to facilitate our analysis we shall impose further nondegeneracy

\footnotetext{
${ }^{1}$ Such subsidiary arguments were also needed in the three-dimensional case.
} 
assumptions on the Newton polyhedron of $P$. In particular, we shall assume that the plane determined by any three vertices of the Newton polyhedron of $P$ does not contain the origin.

In stating our theorems we shall use the following notation. If $V=$ $e s^{a} t^{b} u^{d}$, with $e \neq 0$, with $(a, b, d)$ a vertex of the Newton polyhedron of $P$, and $\Gamma(s, t, u)=(s, t, u, V)=\left(s, t, u, e s^{a} t^{b} u^{d}\right)$, we set

$$
H_{\mathcal{R}}^{V} f(x)=\int_{\mathcal{R}} f(x-\Gamma(s, t, u)) \frac{d s d t d u}{s t u} .
$$

Here $\mathcal{R}$ is a subregion of $\mathbb{R}^{3}$. If $Q=\sum_{j=1}^{k} e_{j} s^{a_{j}} t^{b_{j}} u^{d_{j}}$, with $e_{j} s^{a_{j}} t^{b_{j}} u^{d_{j}}$ running over the monomials of $P$ such that $\left(a_{j}, b_{j}, d_{j}\right)$ lie on an edge of the Newton polyhedron of $P$, we set

$$
H_{\mathcal{R}}^{Q} f(x)=\int_{\mathcal{R}} f(x-\Gamma(s, t, u)) \frac{d s d t d u}{s t u},
$$

where $\Gamma(s, t, u)=(s, t, u, Q(s, t, u))$.

As indicated above, we shall always make the following hypotheses on the Newton polyhedra that we consider:

(H1) No vertex lies on a coordinate hyperplane; that is, if $(a, b, d)$ is a vertex, then $a>0, b>0$ and $d>0$.

(H2) For each edge, if $\left(a_{j}, b_{j}, d_{j}\right)$ lies on that edge, then each of the sequences $\left\{a_{j}\right\},\left\{b_{j}\right\}$ and $\left\{d_{j}\right\}$ is strictly monotonic, and the projection of the line through $\left(a_{j}, b_{j}, d_{j}\right)$ onto a coordinate hyperplane does not pass through the origin.

(H3) The plane determined by any three vertices does not contain the origin.

Remark. In (H2) above, automatically two of the sequences are monotonic in one sense and the third monotonic in the opposite sense.

We shall prove two types of theorem in this paper. We consider a collection of monic monomials $\left\{s^{a_{j}} t^{b_{j}} u^{d_{j}}\right\}$. Let $\mathcal{R}=\{(s, t, u):|s| \leq 1,|t| \leq 1|u| \leq 1\}$. In the first theorem we give a necessary and sufficient condition that $H_{\mathcal{R}}^{P}$ be bounded for the class of all polynomials $P(s, t, u)=\sum e_{j} s^{a_{j}} t^{b_{j}} u^{d_{j}}$ with all $e_{j}$ different from zero. Secondly, with an additional nondegeneracy condition, we give a necessary and sufficient condition that $H_{\mathcal{R}}^{P}$ be bounded for an individual $P$ in terms of the vanishing of certain oscillatory integrals associated to $P$. The condition of the first theorem is expressed solely in terms of the Newton polyhedron while that of the second theorem is expressed in terms of the individual coefficients of the polynomial in question. 
Theorem 1.1. Suppose $\left\{a_{j}, b_{j}, d_{j}\right\}$ are fixed so that hypotheses (H1), (H2) and (H3) are satisfied by the Newton polyhedron generated by the monic monomials $\left\{s^{a_{j}} t^{b_{j}} u^{d_{j}}\right\}$. Then $H_{\mathcal{R}}^{P}$ is bounded on $L^{2}$ for all polynomials $P(s, t, u)=\sum e_{j} s^{a_{j}} t^{b_{j}} u^{d_{j}}$ with all $e_{j}$ different from zero if and only if the following conditions hold:

(i) For each vertex $(a, b, d)$ at least two of $\{a, b, d\}$ are even; and

(ii) If $\left\{\left(a_{l}, b_{l}, d_{l}\right)\right\}$ are the points on any edge of the Newton polyhedron, either all the $a_{l}$ are even, or all the $b_{l}$ are even or all the $d_{l}$ are even.

Before stating our theorem of the second type, some preliminary discussion is in order. First of all it is well-known (see [6]) that if $N(u)$ is any real-valued polynomial of one real variable, then

$$
\int_{-\infty}^{\infty} e^{i N(u)} \frac{d u}{u}
$$

is finite with a bound depending only on the degree of $N$. Now fix a polynomial $P$ in the above class and suppose that $Q(s, t, u)$ is a polynomial corresponding to a bounded edge of the Newton polyhedron generated by the monomials occurring in $P$. Let

$$
\begin{aligned}
& F_{a}^{Q}(x)=\int_{-\infty}^{\infty} \frac{d s}{s}\left\{e^{i x Q(s, 1,1)}+e^{i x Q(s,-1,-1)}-e^{i x Q(s,-1,1)}-e^{i x Q(s, 1,-1)}\right\} \\
& F_{b}^{Q}(x)=\int_{-\infty}^{\infty} \frac{d t}{t}\left\{e^{i x Q(1, t, 1)}+e^{i x Q(-1, t,-1)}-e^{i x Q(-1, t, 1)}-e^{i x Q(1, t,-1)}\right\}
\end{aligned}
$$

and

$$
F_{d}^{Q}(x)=\int_{-\infty}^{\infty} \frac{d u}{u}\left\{e^{i x Q(1,1, u)}+e^{i x Q(-1,-1, u)}-e^{i x Q(-1,1, u)}-e^{i x Q(1,-1, u)}\right\}
$$

Then $F_{a}^{Q}, F_{b}^{Q}$ and $F_{d}^{Q}$ are well defined bounded functions of $x$. Let $\left(a_{1}^{Q}, b_{1}^{Q}, d_{1}^{Q}\right)$ and $\left(a_{2}^{Q}, b_{2}^{Q}, d_{2}^{Q}\right)$ be the two vertices of the bounded edge corresponding to $Q$. Our analysis will show that if $a_{1}^{Q}$ and $a_{2}^{Q}$ are even, then

$$
\int_{0}^{\infty} F_{a}^{Q}(x) \frac{d x}{x}
$$

exists and similarly for the pairs $\left(b_{1}^{Q}, b_{2}^{Q}\right)$ and $\left(d_{1}^{Q}, d_{2}^{Q}\right)$.

The additional hypothesis we require is as follows:

(H4) $P(s, t, u)$ has the form $P=\sum e_{j} s^{a_{j}} t^{b_{j}} u^{d_{j}}$ where the $e_{j}$ are nonzero, and the plane determined by any three points $\left(a_{j_{1}}, b_{j_{1}}, d_{j_{1}}\right),\left(a_{j_{2}}, b_{j_{2}}, d_{j_{2}}\right)$ and $\left(a_{j_{3}}, b_{j_{3}}, d_{j_{3}}\right)$, two of which lie on one edge of the Newton polyhedron of $P$, the third of which lies on another edge of the Newton polyhedron of $P$, does not contain the origin. 
Theorem 1.2. Let $P$ be a polynomial whose Newton polyhedron satisfies (H1), (H2) and (H3) and which also satisfies (H4).

(i) If $H_{\mathcal{R}}^{P}$ is bounded on $L^{2}$, then for all edges, two of the components of each of the two vertices $\left(a_{1}^{Q}, b_{1}^{Q}, d_{1}^{Q}\right)$ and $\left(a_{2}^{Q}, b_{2}^{Q}, d_{2}^{Q}\right)$ generating the edge are even. If $a_{1}^{Q}$ and $a_{2}^{Q}$ are even then

$$
\int_{0}^{\infty} F_{a}^{Q}(x) \frac{d x}{x}=0
$$

Similarly if $b_{1}^{Q}$ and $b_{2}^{Q}$ are even then

$$
\int_{0}^{\infty} F_{b}^{Q}(x) \frac{d x}{x}=0
$$

and if $d_{1}^{Q}$ and $d_{2}^{Q}$ are even then

$$
\int_{0}^{\infty} F_{d}^{Q}(x) \frac{d x}{x}=0
$$

(ii) Conversely, if for each $Q$, at least two components of $\left\{a_{1}^{Q}, b_{1}^{Q}, d_{1}^{Q}\right\}$ and $\left\{a_{2}^{Q}, b_{2}^{Q}, d_{2}^{Q}\right\}$ are even, and if $a_{1}^{Q}$ and $a_{2}^{Q}$ are even and

$$
\int_{0}^{\infty} F_{a}^{Q}(x) \frac{d x}{x}=0
$$

if $b_{1}^{Q}$ and $b_{2}^{Q}$ are even and

$$
\int_{0}^{\infty} F_{b}^{Q}(x) \frac{d x}{x}=0
$$

and if $d_{1}^{Q}$ and $d_{2}^{Q}$ are even and

$$
\int_{0}^{\infty} F_{d}^{Q}(x) \frac{d x}{x}=0
$$

then $H_{\mathcal{R}}^{P}$ is bounded on $L^{2}$.

Remark. If the necessary conditions that two of $\left\{a_{1}^{Q}, b_{1}^{Q}, d_{1}^{Q}\right\}$ and that two of $\left\{a_{2}^{Q}, b_{2}^{Q}, d_{2}^{Q}\right\}$ are even are satisfied, it follows that both $a_{1}^{Q}$ and $a_{2}^{Q}$ are even or both $b_{1}^{Q}$ and $b_{2}^{Q}$ are even or both $d_{1}^{Q}$ and $d_{2}^{Q}$ are even. Therefore if say both $a_{1}^{Q}$ and $a_{2}^{Q}$ are even and both $b_{1}^{Q}$ and $b_{2}^{Q}$ are even and if $\int_{0}^{\infty} F_{a}^{Q}(x) \frac{d x}{x}=0$, then also we have $\int_{0}^{\infty} F_{b}^{Q}(x) \frac{d x}{x}=0$.

The proof of the necessity in these theorems will proceed by a dilation argument which requires us to analyze global variants of our theorems in certain special cases. See Sections 7 and 8. 
The organization of this paper is as follows. In Section 2 we make some preliminary estimates which the reader may wish to refer back to later. In Section 3 we establish that a large portion of the Fourier multiplier for the operators in our main theorems is under control, using methods of wide applicability which do not use the most essential properties of the Newton polyhedron. In Section 4 we return to the full operators and reduce matters to the skeleton of the Newton polyhedron, that is the vertices and edges. In Section 5, we show boundedness of edge operators imply the boundedness in Theorems 1.1 and 1.2. In Section 6, we make a reduction in the study of edge operators which in particular proves the sufficiency in Theorem 1.1. In Section 9 we conclude the sufficiency part of Theorem 1.2. Many of the arguments of this section echo those of Section 8. Sections 10 and 11 are devoted to the proof of the necessity parts of Theorems 1.1 and 1.2. Finally, in Section 12 we give two examples. One shows that the cancellation conditions in Theorem 1.2 do indeed depend upon the coefficients of the polynomial in question. The other example is a Newton polyhedron all of whose vertices have even coordinates but for which every choice of coefficients in the polynomial gives rise to an unbounded operator.

After the submission of this manuscript, we learned of a related paper Triple Hilbert transforms along polynomial surfaces by Yong-Kum Cho, Sunggeum Hong, Joonil Kim and Chan Woo Yang, written at the same time as ours. They prove an analogue of Theorem 1.1 in which they also obtain $L^{p}$ estimates.

\section{Estimates for certain sublevel sets and oscillatory integrals}

We begin with a sublevel set estimate.

Lemma 2.1. Let

$$
P(u)=a_{1} u+\cdots+a_{n} u^{n},
$$

$a_{j}$ real, be a polynomial with no constant term. Suppose $J$ is a subinterval of the positive reals such that $P(u)$ and $P^{(j)}(u), 1 \leq j \leq n$, are of constant sign and monotonic on $J$. Suppose in addition for some $j_{0}, 1 \leq j_{0} \leq n$, and all $u \in J$

$$
\left|u^{j_{0}} P^{\left(j_{0}\right)}(u)\right| \geq\left|u^{j} P^{(j)}(u)\right|, \quad 1 \leq j \leq n .
$$

Assume further that for some subinterval $[a, b]$ of $J$ with $b \leq C(n) a$

$$
\alpha \leq|P(u)| \leq \beta
$$

for all $u, a \leq u \leq b$. 
Then there exists a constant $A(n)$ such that

$$
\frac{b-a}{a} \leq A(n)\left(\frac{\beta-\alpha}{\alpha}\right)^{1 / j_{0}} .
$$

A similar result holds if $J$ is contained in the negative reals.

Proof. The proof relies on ideas from the Proof of Theorem 3.1 of [2].

$$
u^{j} P^{(j)}(u)=\sum_{\ell=j}^{n} a_{\ell} C(\ell, j) u^{\ell}
$$

with

$$
C(\ell, j)=\frac{\ell !}{(\ell-j) !} .
$$

We claim that for all $u$ in $[a, b]$,

$$
\left|P^{\left(j_{0}\right)}(u)\right| \geq A_{0}(n) \frac{\alpha}{a^{j_{0}}} .
$$

In fact, suppose for some $u \in[a, b]$ and all $1 \leq j \leq n$,

$$
\left|u^{j} P^{(j)}(u)\right|<\eta
$$

for some positive $\eta$. Then by Cramér's rule, we see that for each $\ell, 1 \leq \ell \leq n$.

$$
\left|a_{\ell} u^{\ell}\right| \leq A_{1}(n) \eta .
$$

This implies

$$
|P(u)| \leq A_{2}(n) \eta
$$

In view of (2.1) we see that if $\left|u^{j_{0}} P^{\left(j_{0}\right)}(u)\right|<|t| \geq \frac{1}{\left|\xi_{2}\right|} \eta$ for some $u \in J$,

$$
|P(u)| \leq A_{2}(n) \eta
$$

But we are assuming

$$
|P(u)| \geq \alpha
$$

for all $u$ in $[a, b]$. Thus for all $u$ in $[a, b]$,

$$
u^{j_{0}}\left|P^{\left(j_{0}\right)}(u)\right| \geq \frac{\alpha}{A_{2}(n)} .
$$

Then (2.3) follows since $b \leq C(n) a$. We shall see that (2.4) implies

$$
\left|P^{\prime}(u)\right| \geq \gamma(n) \frac{\alpha(b-a)^{j_{0}-1}}{a^{j_{0}}}
$$


for some $\gamma(n)>0$ and all $u$ in a subinterval $\left[a^{\prime}, b^{\prime}\right]$ of $[a, b]$ with $b^{\prime}-a^{\prime} \geq \frac{b-a}{2^{j_{0}}}$. Let us see that (2.5) implies the conclusion of Lemma 2.1. Since $P^{\prime}(u)$ and $P(u)$ are of constant sign and monotonic on $[a, b]$

$$
\beta-\alpha \geq\left|P\left(b^{\prime}\right)-P\left(a^{\prime}\right)\right|=\int_{a^{\prime}}^{b^{\prime}}\left|P^{\prime}(u)\right| d u \geq \frac{\gamma(n) \alpha|b-a|^{j_{0}}}{2^{j_{0}} a^{j_{0}}} .
$$

So

$$
\frac{b-a}{a} \leq 2\left(\frac{1}{\gamma(n)}\right)^{1 / j_{0}}\left(\frac{\beta-\alpha}{\alpha}\right)^{1 / j_{0}},
$$

and the lemma is proved.

We have to see that (2.4) implies (2.5). To see this it suffices to show that if for some $\ell$

$$
\left|P^{(\ell)}(u)\right| \geq \lambda
$$

on a subinterval $[c, d]$ of $J$, then

$$
\left|P^{(\ell-1)}(u)\right| \geq \frac{1}{2} \lambda(d-c)
$$

on a subinterval $\left[c^{\prime}, d^{\prime}\right]$ of $[c, d]$ with $\left|d^{\prime}-c^{\prime}\right| \geq \frac{1}{2}|d-c|$.

We may assume without loss of generality that $P^{(\ell)}(u) \geq 0$ on $[c, d]$. If $P^{(\ell-1)}(u) \geq 0$ on $[c, d]$

$$
\begin{aligned}
P^{(\ell-1)}(u)-P^{(\ell-1)}(c) & \geq \int_{c}^{u} P^{(\ell)}(u) d u \\
& \geq \lambda(u-c) .
\end{aligned}
$$

so if $u>\frac{d+c}{2}, P^{(\ell-1)}(u)>\frac{\lambda}{2}(d-c)$, and we can take $c^{\prime}=\frac{d+c}{2}$ and $d^{\prime}=d$. If $P^{(\ell-1)}(u) \leq 0$ on $[c, d]$

$$
P^{(\ell-1)}(d)-P^{(\ell-1)}(u)>\lambda(d-u) .
$$

So $\left|P^{(\ell-1)}(u)\right|>\frac{1}{2} \lambda(d-c)$ for $c \leq u \leq \frac{d+c}{2}$. This concludes the proof of Lemma 2.1.

The purpose of the next lemma is to deal with the assumption that $b \leq C(n) a$ in Lemma 2.1 .

Lemma 2.2. Let $P(u)$ be a polynomial of degree $n$ with $P(0)=0$. Then there exist at most $A(n)$ intervals $I_{\ell}, 1 \leq \ell \leq A(n)$, in the positive reals, each of the form $I_{\ell}=(a(\ell), b(\ell))$ with $b(\ell) \leq C(n) a(\ell)$ such that for $u$ in the complement of the union of the $I_{\ell},|1+P(u)|>1 / 4$. A similar conclusion holds for the negative reals. 
Proof. Let $P(u)=\sum_{j=1}^{n} a_{j} u^{j}$. For each $j$ and $k \neq j$, let

$$
E_{j, k}=\left\{u: \frac{1}{2 n} \leq \frac{\left|a_{j} u^{j}\right|}{\left|a_{k} u^{k}\right|} \leq 2 n\right\} .
$$

Then $\cup E_{j, k}$ is a union of at most $n^{2}$ intervals, $(a(\ell), b(\ell)), 1 \leq \ell \leq n^{2}$ with $b(\ell) \leq c(n) a(\ell)$. In the complement of $\cup_{k} E_{j, k}$,

$$
\frac{1}{2}\left|a_{j} u^{j}\right| \leq|P(u)| \leq \frac{5}{4}\left|a_{j} u^{j}\right| .
$$

Thus for $u$ is in the complement of $\cup_{k} E_{j, k}$ with $|1+P(u)|<\frac{1}{4}$, then $\frac{3}{4} \leq|P(u)| \leq 5 / 4,\left|a_{j} u^{j}\right| \geq \frac{1}{2}$ and $\left|a_{j} u^{j}\right| \leq 3$. That is $\frac{1}{2\left|a_{j}\right|} \leq u^{j} \leq \frac{3}{\left|a_{j}\right|}$. Thus if we add the $n$ intervals $\left[\left(\frac{1}{2\left|a_{j}\right|}\right)^{1 / j},\left(\frac{3}{\left|a_{j}\right|}\right)^{1 / j}\right]$ to the $n^{2}$ intervals $E_{j, k}$, we have the required intervals.

Our estimates for oscillatory integrals depend on Corollary 3.6 of [2] which we state here for the convenience of the reader.

Corollary 3.6 of [2]. Let $a_{1}, \ldots, a_{N} \in \mathbb{R}$. Suppose $a_{\ell} \neq 1$ for $\ell=$ $1,2, \ldots, N$. For $\lambda \geq 1$ and $B \geq 1$, consider

$$
I(\lambda)=\int_{1}^{B} \exp \left\{i \lambda s+y_{1} s^{a_{1}}+\cdots+y_{N} s^{a_{N}}\right\} \frac{d s}{s} .
$$

Then

$$
|I(\lambda)| \leq C \lambda^{-1 /(N+1)} .
$$

Here $C=C\left(a_{1}, \ldots, a_{N}\right)$ is independent of $B, y_{1}, \ldots, y_{N}$.

In our next lemma, $P(s, u)$ is a polynomial of degree $n$ with no linear term, and $L$ is a subinterval of $[A, \infty]$, where $A>1$. The interval $L$ can depend on $u$, but $A$ must be independent of $u$. We consider

$$
I(L)=\int_{L} e^{i(s+P(s, u))} \frac{d s}{s} .
$$

Lemma 2.3. With $P$ and $L$ as above there are positive $\eta_{1}$ and $\eta_{2}$ and a set $E$ of u's so that for $u$ not in $E$

$$
|I(L)| \leq C_{1}\left(\eta_{1}, \eta_{2}, n\right) A^{-\eta_{1}},
$$

and $E$ is contained in a bounded number of intervals $K_{j}$

$$
K_{j}=\left[a_{j}, a_{j}+C_{2}\left(\eta_{1}, \eta_{2}, n\right) A^{-\eta_{2}} a_{j}\right] .
$$

Also the number of intervals $K_{j}$ depends only on $n$. A similar result holds if $L \subset(-\infty,-A)$. 
Proof. We may assume $L=[A, B]$, with $B$ perhaps depending on $u$. If $P(s, u)$ has no term linear in $s$, Lemma 2.3 follows from Corollary 3.6 of [2] after making a change of variables $s=A \sigma$. Otherwise

$$
P(s, u)=s P_{1}(u)+P_{2}(s, u)
$$

where $P_{1}$ has no constant term and no monomial of $P_{2}$ is linear in $s$. We now change variables setting $s=\sigma A$. Then

$$
I=\int_{1}^{B^{\prime}} e^{i A s\left(1+P_{1}(u)\right)+P_{2}(A s, u)} \frac{d s}{s} .
$$

We let $E=\left\{u:\left|1+P_{1}(u)\right|<\frac{1}{\sqrt{A}}\right\}$. For $u$ in the complement of $E$, the estimate (2.6) follows from Corollary 3.6 of [2]. Using 2.2, we can divide the set of $u$ 's in $E$ into a bounded number of intervals in which the hypotheses of Lemma 2.1 apply. Then applying Lemma 2.1, we obtain the desired conclusion on the set $E$.

Lemma 2.4. Let $P(s, t, u)$ be a polynomial such that each monomial of $P$ contains a positive power of $s$ and a positive power of $u$. For $t \geq 1$, let

$$
I(t)=\int_{u \geq t} \frac{d u}{u}\left|\int_{|s| \in L_{1}(u, t)} \frac{d s}{s} e^{i(s+P(s, t, u))}\right|,
$$

where $L_{1}$ is a subinterval of $(u, \infty)$. For any $\delta>0$ and $0<t<1$, let

$$
J(t)=\int_{0<u \leq t} \frac{d u}{u}\left|\int_{|s| \in L_{2}(u, t)} \frac{d s}{s} e^{i(s+P(s, t, u))}\right|
$$

where $L_{2}$ is a subinterval of $\left[u^{-\delta}, \infty\right)$. Then for some positive $\eta_{1}$ and $\eta_{2}=\eta_{2}(\delta)$

$$
I(t) \leq \frac{C(n)}{t^{\eta_{1}}}
$$

and

$$
J(t) \leq C(n, \delta) t^{\eta_{2}(\delta)}
$$

Proof. Consider the estimate for $I(t)$. We divide the $u$ integration into dyadic intervals $2^{k} \leq u<2^{k+1}$, with $k \geq \log |t|-1$. For $u$ in the interval $\left[2^{k}, 2^{k+1}\right], L_{1}(u, t) \subset\left(2^{k}, \infty\right)$. We apply Lemma 2.3. For $u$ not in $E$, the $s$ integral is at most $2^{-\eta_{1} k}$, for some $\eta_{1}>0$ and so

$$
\int_{\substack{2^{k} \leq u \leq 2^{k+1} u \notin E \\ u \notin}} \frac{d u}{u}\left|\int_{|s| \in L_{1}(u, t)} e^{i(s+P(s, t, u))} \frac{d s}{s}\right| \leq C 2^{-\eta_{1} k} .
$$


Since the $s$ integral is uniformly bounded (see for example [6]),

$$
\int_{\substack{u \in E \\ 2^{k} \leq u \leq 2^{k+1}}} \frac{d u}{u}\left|\int_{|s| \in L_{1}(u, t)} e^{i[s+P(s, t, u)]} \frac{d s}{s}\right| \leq C \sup _{\substack{a \\ 2^{k} \leq a \leq 2^{k+1}}} \int_{a}^{a+C 2^{-\eta_{2} k} a} \frac{d u}{u} \leq C 2^{-\eta_{2} k} .
$$

Summing on $k$, we arrive at the estimate for $I(t)$. The estimate for $J(t)$ is obtained in a similar manner.

The next lemma gives a basic estimate for an oscillatory integral which we will find very useful on several occasions.

Lemma 2.5. Let

$$
E(s)=\sum_{j=1}^{k} e_{j} s^{\ell_{j}} \quad \text { and } \quad F(s)=\sum_{j=1}^{n} f_{j} s^{m_{j}}
$$

with the $e_{j}$ and $f_{j}$ not zero. Assume the $\ell_{j}$ and $m_{j}$ are strictly increasing.

For any $B>0$ set

$$
I(\lambda)=\int_{0}^{B} e^{i \lambda E(s)} \sin \lambda F(s) \frac{d s}{s} .
$$

Then if $\ell_{k}>m_{n} \geq 1$

$$
|I(\lambda)| \leq C \lambda^{\delta}
$$

for some $\delta>0$. If $1 \leq \ell_{1}<m_{1}$

$$
|I(\lambda)| \leq C \lambda^{-\delta} \quad \text { for some } \quad \delta>0 .
$$

Here, $C$ may depend on the $e_{j}$ and $f_{j}$.

Proof. In this lemma we are mainly interested in small $\lambda>0$ in (2.9) and large $\lambda>0$ in (2.10). To prove (2.9) we write

$$
I(\lambda)=\int_{0 \leq s \leq A}+\int_{A \leq s \leq u_{0}}+\int_{u_{0} \leq s \leq B}:=I_{1}+I_{2}+I_{3}
$$

where $A>1$ is to be chosen as a function of the $\left\{e_{j}\right\}$ and $\left\{f_{j}\right\}$, and $u_{0}=$ $\left(\frac{1}{\lambda}\right)^{\frac{1}{m_{n}}-\epsilon}$, where $\epsilon>0$ will be chosen small. For any fixed $A$,

$$
\left|I_{1}\right| \leq C(A) \lambda
$$

For $A>1$

$$
\left|I_{2}\right| \leq C \lambda \int_{1}^{u_{0}} s^{m_{n}} \frac{d s}{s} \leq C \lambda^{\epsilon m_{n}}
$$


If $A$ is sufficiently large and $s>A$,

$$
\left|\frac{d}{d s}(E(s) \pm F(s))\right| \geq C s^{\ell_{k}-1}
$$

for some positive $C$, so

$$
\left|I_{3}\right| \leq \frac{C}{\lambda} \int_{u_{0}}^{\infty} \frac{d s}{s^{l_{k}+1}}
$$

Thus

$$
\left|I_{3}\right| \leq \frac{C}{\lambda} \lambda^{\frac{\ell_{k}}{m_{n}}-\epsilon \ell_{k}} \leq C \lambda^{\delta}
$$

if $\epsilon$ is sufficiently small since $\ell_{k}>m_{n}$. This concludes the proof of (2.9).

To prove (2.10), we write

$$
I(\lambda)=\int_{0}^{u_{0}}+\int_{u_{0}}^{A}+\int_{A}^{B}=I_{1}+I_{2}+I_{3}
$$

where

$$
u_{0}=\left(\frac{1}{\lambda}\right)^{\frac{1}{m_{1}}+\epsilon}
$$

with

$$
\frac{1}{m_{1}}+\epsilon \leq \frac{1}{\ell_{1}}-\epsilon
$$

and $A$ is sufficiently small. For any fixed $A$,

$$
\left|I_{3}\right| \leq C \lambda^{-\delta}
$$

for some $\delta>0$ by van der Corput's lemma. (Not all the coefficients of $E \pm F$ can be zero.) If $u_{0}<1$,

$$
\left|I_{1}\right| \leq C \lambda u_{0}^{m_{1}} \leq C \lambda^{-m_{1} \epsilon}
$$

For $A$ sufficiently small, and $0 \leq s \leq A$,

$$
\left|\frac{d}{d s}(E(s) \pm F(s))\right| \geq C s^{\ell_{1}-1} .
$$

So

$$
\left|I_{2}\right| \leq \frac{C}{\lambda u_{0}^{\ell_{1}}}+\frac{C}{\lambda} \int_{u_{0}}^{A} \frac{d s}{s^{\ell_{1}+1}} \leq \frac{C}{\lambda u_{0}^{\ell_{1}}} \leq \frac{C}{\lambda} \lambda^{\left(\frac{\ell_{1}}{m_{1}}+\epsilon \ell_{1}\right)} \leq \frac{C}{\lambda} \lambda^{\ell_{1}\left(\frac{1}{m_{1}}-\epsilon\right)} \leq C \lambda^{-\ell_{1} \epsilon} .
$$

This finishes the proof of Lemma 2.5.

Our final lemma in Section 2 concerns double integrals. This lemma is closely related to work of Patel, [3]. 
Lemma 2.6. Let

$$
Q(t, u)=\sum_{\ell=1}^{L} e_{\ell} t^{b_{\ell}} u^{d_{\ell}}
$$

be a real valued polynomial in two real variables. Assume none of the $e_{\ell}, b_{\ell}$ and $d_{\ell}$ are zero, and that the sequences $\left\{b_{\ell}\right\}$ and $\left\{d_{\ell}\right\}$ are strictly monotonic. Furthermore, suppose the points $\left(b_{\ell}, d_{\ell}\right)$ lie on a line not passing through the origin. Finally assume at least one of $b_{1}$ and $d_{1}$ are even and at least one of $b_{L}$ and $d_{L}$ are even.

For a given pair of positive parameters $\alpha$ and $\beta$, set

$$
\Delta=\left\{(t, u):|t| \leq A, \quad|u| \leq B, \quad A^{\prime} \leq|t|^{\alpha}|u|^{\beta} \leq B^{\prime}\right\} .
$$

Set

$$
\begin{aligned}
I & =\int_{\Delta} e^{i Q(t, u)} \frac{d t d u}{t u}, \\
I_{\tau} & =\int_{\Delta \cap\{t:|t| \leq \tau\}} e^{i Q(t, u)} \frac{d t d u}{t u}, \\
I^{v} & =\int_{\Delta \cap\{u:|u| \geq v\}} e^{i Q(t, u)} \frac{d t d u}{t u},
\end{aligned}
$$

and

$$
I_{\tau}^{v}=\int_{\Delta \cap\{(t, u):|t| \leq \tau,|u| \leq v\}} e^{i Q(t, u)} \frac{d t d u}{t u} .
$$

Then

(i) $|I| \leq C$,

(ii) $\left|I_{\tau}\right| \leq C \tau^{\delta}$ for some $\delta>0$,

(iii) $\left|I^{v}\right| \leq C \frac{1}{v^{\delta}}$ for some $\delta>0$, and

(iv) $\left|I_{\tau}^{v}\right| \leq C \tau^{\delta}$ for some $\delta>0$.

Here, $C$ may depend on the coefficients of $Q$, but not on $A, B, A^{\prime}$ or $B^{\prime}$.

Proof. The proof of all parts of Lemma 2.6 are similar, and we will content ourselves with the proof of (iii).

Let $\mathcal{R}_{p, q}$ be that portion of $\Delta$ for which $2^{-p} \leq|t| \leq 2 \cdot 2^{-p}$ and $2^{-q} \leq$ $|u| \leq 2 \cdot 2^{-q}$, and let $I(p, q)$ be the contribution to $I^{v}$ from $\mathcal{R}_{p, q}$. So

$$
I^{v}=\sum_{p \in \mathbb{Z}} \sum_{q \in \mathbb{Z}, 2^{-q} \geq v} I(p, q) .
$$


Let

$$
U(p, q)=\int_{\mathcal{R}_{p, q}} \exp i\left(e_{L} t^{b_{L}} u^{d_{L}}\right) \frac{d t d u}{t u}
$$

and

$$
V(p, q)=\int_{\mathcal{R}_{p, q}} \exp i\left(e_{1} t^{b_{1}} u^{d_{1}}\right) \frac{d t d u}{t u} .
$$

Our assumption that at least one of $b_{L}$ and $d_{L}$ is even implies $U(p, q)=0$, and our assumption that at least one of $b_{1}$ and $d_{1}$ is even implies $V(p, q)=0$. We shall divide the $(p, q)$ into two sets $S_{1}$ and $S_{2}$. We shall prove

$$
\sum_{\substack{(p, q) \in S_{1} \\ 2-q \geq v}}|I(p, q)-U(p, q)| \leq C / v^{\delta}
$$

and

$$
\sum_{\substack{(p, q) \in S_{2} \\ 2^{-q} \geq v}}|I(p, q)-V(p, q)| \leq C / v^{\delta} .
$$

This will then prove the lemma.

It is convenient to write $\sigma_{\ell}=\left(b_{\ell}, d_{\ell}\right), r=(p, q)$ and $w=\sigma_{1}-\sigma_{L}$. Put $S_{1}=\{r: r \cdot w \geq 0\}$ and $S_{2}=\{r: r \cdot w \leq 0\} . S_{1}$ essentially corresponds to the region where $|t|^{b_{L}}|u|^{d_{L}} \geq|t|^{b_{1}}|u|^{d_{1}}$. We will only consider the estimate (2.11). The estimate for (2.12) is similar. Changing variables we see

$$
I(p, q)=\int_{\overline{\mathcal{R}}_{p, q}} \int \exp \left(i \sum_{\ell=1}^{L} e_{\ell} 2^{-p b_{\ell}} 2^{-q d_{\ell}} t^{b_{\ell}} u^{d_{\ell}}\right) \frac{d t d u}{t u}
$$

and

$$
U(p, q)=\int_{\overline{\mathcal{R}}_{p, q}} \int \exp \left(i e_{L} 2^{-p b_{L}} 2^{-q d_{L}} t^{b_{L}} u^{d_{L}}\right) \frac{d t d u}{t u}
$$

where

$$
\overline{\mathcal{R}}(p, q)=\left\{(t, u): 1 \leq|t| \leq 2,1 \leq|u| \leq 2, A^{\prime}(p, q) \leq|t|^{\alpha}|u|^{\beta} \leq B^{\prime}(p, q)\right\} .
$$

Thus we have two estimates

$$
|I(p, q)-U(p, q)| \leq \frac{D}{\left(2^{-p b_{L}} 2^{-q d_{L}}\right)^{\eta}}
$$

and

$$
\begin{aligned}
|I(p, q)-U(p, q)| \leq C \sup _{\substack{\ell \leq \ell \leq L-1 \\
1 \leq \ell-q d_{L}}}\left(2^{-p b_{\ell}} 2^{-q d_{\ell}}\right)^{\eta} \\
\leq C\left(2^{-p b_{L}} 2^{-q d_{L}}\right)^{\eta}
\end{aligned}
$$

for some $\eta>0$. 
We first sum over all $(p, q)$ with $r \cdot w=N$ and $2^{-q} \geq v$ and then sum over $N$. Since $r \cdot w=N$ we have

$$
p\left(b_{1}-b_{L}\right)+q\left(d_{1}-d_{L}\right)=N
$$

or

$$
p=\frac{N}{b_{1}-b_{L}}+q \frac{d_{1}-d_{L}}{b_{1}-b_{L}} .
$$

Then (2.13) and (2.14) become

$$
|I(p, q)-U(p, q)| \leq C\left(2^{\gamma N} 2^{-\alpha q}\right)^{-\eta}
$$

and

$$
|I(p, q)-U(p, q)| \leq C\left(2^{(\gamma-\kappa) N} 2^{-\alpha q}\right)^{\eta}
$$

where $\gamma=-\frac{b_{L}}{b_{1}-b_{L}}, \kappa>0$ and $\alpha \neq 0$ since the line through the $\left(b_{\ell}, d_{\ell}\right)$ does not go through the origin. Let us assume $\alpha>0$. If we sum on $q$ with $\alpha q \geq\left(\gamma-\frac{\kappa}{2}\right) N$, using (2.16), we get a contribution of $2^{-N \eta \kappa / 2}$. If we use (2.15) for $q$ with $\alpha q \leq\left(\gamma-\frac{\kappa}{2}\right) N$, we get another contribution of $2^{-N \kappa \eta / 2}$. Thus

$$
\sum_{r, r \cdot w=N}|I(p, q)-U(p, q)| \leq C 2^{-\frac{\eta}{2} N \kappa} .
$$

This gives the desired result if $2^{N}>v^{\epsilon}$ for any positive $\epsilon$. If $|v|>\frac{1}{2^{N / \epsilon}}$, we use only (2.16), and find

$$
\sum_{r, r \cdot w=N}|I(p, q)-U(p, q)| \leq C \frac{2^{(\gamma-\kappa) N}}{|v|^{\alpha}}
$$

If $\epsilon>0$ is sufficiently small we can now sum in $N$. This completes the proof of the lemma.

\section{Estimates for some triple integrals}

The purpose of this section is to estimate some triple integrals which will in effect show pieces of the multiplier

$$
m\left(\xi_{1}, \xi_{2}, \xi_{3}, \xi_{4}\right)=\int \exp \left(i \xi_{1} s+i \xi_{2} t+i \xi_{3} u+i \xi_{4} P(s, t, u)\right) \frac{d s d t d u}{s t u}
$$

are bounded. In this section, we shall assume that the coefficient of each power of $u$ is a monomial in $s$ and $t$. Moreover we assume each monomial of $P$ has a positive power of each variable. All estimates are to be uniform in the coefficients of $P$. 
For a given set of positive parameters $\left\{\alpha_{i}, \beta_{i}, \gamma_{i}, \bar{\alpha}_{i}, \bar{\beta}_{i}, \bar{\gamma}_{i}\right\}$, let $\Delta$ denote the following region in $\mathbb{R}^{3}$,

$$
\Delta=\left\{(s, t, u):|s|^{\alpha_{i}}|t|^{\beta_{i}}|u|^{\gamma_{i}} \leq A_{i}|s|^{\bar{\alpha}_{i}}|t|^{\bar{\beta}_{i}}|u|^{\bar{\gamma}_{i}}, 1 \leq i \leq N\right\} .
$$

Here $\Delta$ and in particular the constants $A_{i}$ will change from place to place as we make changes of variables, but our estimates will be uniform in the $A_{i}$. For a fixed $N$, all estimates are to be uniform. We let

$$
\begin{aligned}
& \mathcal{R}_{1}=\left\{(s, t, u):|s| \geq \frac{1}{\left|\xi_{1}\right|},|t| \geq \frac{1}{\left|\xi_{2}\right|},|u| \geq \frac{1}{\left|\xi_{3}\right|}\right\}, \\
& \mathcal{R}_{2}=\left\{(s, t, u):|s| \geq \frac{1}{\left|\xi_{1}\right|},|t| \geq \frac{1}{\left|\xi_{2}\right|},|u| \leq \frac{1}{\left|\xi_{3}\right|}\right\}, \\
& \mathcal{R}_{3}=\left\{(s, t, u):|s| \leq \frac{1}{\left|\xi_{1}\right|},|t| \geq \frac{1}{\left|\xi_{2}\right|},|u| \leq \frac{1}{\left|\xi_{3}\right|}\right\} .
\end{aligned}
$$

Let

$$
\begin{aligned}
& J_{1}=\int_{\mathcal{R}_{1} \cap \Delta} e^{i \xi_{1} s} e^{i \xi_{2} t} e^{i \xi_{3} u} e^{i \xi_{4} P(s, t, u)} \frac{d s d t d u}{s t u}, \\
& J_{2}=\int_{\mathcal{R}_{2} \cap \Delta} e^{i \xi_{1} s} e^{i \xi_{2} t} e^{i \xi_{4} P(s, t, u)} \frac{d s d t d u}{s t u}, \\
& J_{3}=\int_{\mathcal{R}_{2} \cap \Delta}\left(1-e^{i \xi_{3} u}\right) e^{i \xi_{1} s} e^{i \xi_{2} t} e^{i \xi_{4} P(s, t, u)} \frac{d s d t d u}{s t u}, \\
& J_{4}=\int_{\mathcal{R}_{3} \cap \Delta}\left(1-e^{i \xi_{3} u}\right) e^{i \xi_{1} s} e^{i \xi_{2} t} e^{i \xi_{4} P(s, t, u)} \frac{d s d t d u}{s t u}, \\
& J_{5}=\int_{\mathcal{R}_{3} \cap \Delta}\left(1-e^{i \xi_{1} s}\right) e^{i \xi_{4} P(s, t, u)} e^{i \xi_{2} t} \frac{d s d t d u}{s t u} .
\end{aligned}
$$

Lemma 3.1. For $\ell=1,2,3,4$ and 5

$$
\left|J_{\ell}\right| \leq C
$$

where $C$ is independent of the coefficients of $P$.

Remark. Lemma 3.1 effectively reduces the analysis of the Fourier multiplier to the study of two types of integral, the first of the form

$$
\int_{\mathcal{R}_{3} \cap \Delta} e^{i \xi_{2} t} e^{i \xi_{4} P(s, t, u)} \frac{d s d t d u}{s t u}
$$

and the second of the form

$$
\int_{\mathcal{R} \cap \Delta} e^{i \xi_{1} s} e^{i \xi_{2} t} e^{i \xi_{3} u} e^{i \xi_{4} P(s, t, u)} \frac{d s d t d u}{s t u}
$$

where

$$
\mathcal{R}=\left\{(s, t, u):|s| \leq \frac{1}{\left|\xi_{1}\right|},|t| \leq \frac{1}{\left|\xi_{2}\right|},|u| \leq \frac{1}{\left|\xi_{3}\right|}\right\} .
$$


Proof. In each integral we may make a change of variables so that $\xi_{1}=$ $\xi_{2}=\xi_{3}= \pm 1$. Without loss of generality we take the case of + signs. To bound $J_{1}$ we divide the $s, t, u$ integration into six regions according to the size of the variables. For example in one region we have $|s| \geq|t| \geq|u| \geq 1$. The estimate for $J_{1}$ then follows from the estimate for $I(t)$ in Lemma 2.4.

We next consider $J_{2}$. We make a change of variables in the $u$ integral so that

$$
\xi_{4} P(s, t, u)=\sum_{j} e_{j}(\xi) s^{a_{j}} t^{b_{j}} u^{d_{j}}
$$

where, for at least one $j_{0},\left|e_{j_{0}}(\xi)\right|=1$ and $\left|e_{j}(\xi)\right| \leq 1$ for all $j$. We now perform the $u$ integral first. Then by putting $v=u^{d_{j_{0}}}$ and applying Corollary 3.6 of [2] we see the contribution from $|u| \geq 1$ is at most $C(N) /|s t|^{\eta}$ for some positive $\eta$. So this contribution is bounded. For a sufficiently large $\rho$, the contribution from

$$
|u| \leq \frac{1}{(\max (|t|,|s|))^{\rho}}
$$

is clearly bounded. So it remains to consider

$$
\int_{\mathcal{R}_{4} \cap \Delta} e^{i s} e^{i t} e^{i P(s, t, u)} \frac{d s d t d u}{s t u}
$$

where

$$
\mathcal{R}_{4}=\left\{(s, t, u):|s| \geq 1,|t| \geq|s|, \quad \frac{1}{|t|^{\rho}} \leq|u| \leq 1\right\}
$$

plus a similar integral where $|s| \geq|t|$. In the integral over $\mathcal{R}_{4} \cap \Delta$ we shall integrate first with respect to $t$, then $u$, then $s$. Thus this integral becomes the sum of two integrals

$$
\iiint_{\mathcal{R}_{5} \cap \Delta} e^{i s} e^{i t} e^{i P(s, t, u)} \frac{d t}{t} \frac{d s}{s} \frac{d u}{u}
$$

and

$$
\iiint_{\mathcal{R}_{6} \cap \Delta} e^{i s} e^{i t} e^{i P(s, t, u)} \frac{d t}{t} \frac{d s}{s} \frac{d u}{u}
$$

where

$$
\mathcal{R}_{5}=\left\{(s, t, u):|s| \geq 1,1 \geq|u| \geq \frac{1}{|s|^{\rho}},|t| \geq|s|\right\}
$$

and

$$
\mathcal{R}_{6}=\left\{(s, t, u):|s| \geq 1,0 \leq|u| \leq \frac{1}{|s|^{\rho}},|t| \geq \frac{1}{|u|^{1 / \rho}}\right\}
$$


To deal with the integral over $\mathcal{R}_{5} \cap \Delta$ we divide the $s$ integration into dyadic intervals $L_{k}=\left\{s: 2^{k} \leq|s| \leq 2^{k+1}\right\}$. Fix $s$. We use Lemma 2.3 to see that for $u$ outside a bounded number of exceptional intervals of the form $E_{a}=\left\{u: a \leq|u| \leq a+a c 2^{-\eta_{1} k}\right\}$, the $t$ integral is at most $2^{-\eta_{2} k}$. Thus the $t, u$ integral is at most $C k 2^{-\eta_{2} k}$. So

$$
\sum_{k} \int_{s \in L_{k}} \int_{\left\{1 /|s|^{\rho} \leq|u| \leq 1\right\} \backslash \cup_{a} E_{a}} \int \cdots \frac{d t d u d s}{t u s}
$$

is bounded. On the other hand,

$$
\int_{|s| \leq|t|<\infty} e^{i(t+P(s, t, u))} \frac{d t}{t}
$$

is uniformly bounded. So

$$
\sum_{k} \int_{s \in L_{k}} \int_{u \in \cup_{a} E_{a}} \int_{|s| \leq|t|<\infty} \cdots \frac{d t d u d s}{t u s}
$$

is uniformly bounded. The boundedness of the integral over $\mathcal{R}_{6} \cap \Delta$ follows from the estimate for $J$ in Lemma 2.4 .

To see that $J_{3}$ is bounded we note that Lemma 2.4 implies

$$
\left|\iint_{\{|s| \geq 1,|t| \geq 1\} \cap \Delta} e^{i s} e^{i t} e^{i \xi_{4} P(s, t, u)} \frac{d s d t}{s t}\right| \leq C
$$

uniformly in $u$.

To estimate $J_{4}$, we note that we may replace $e^{i s}$ by 1 (Recall we normalized so that $\xi_{1}=1$ ) and the one dimensional $t$ integral is uniformly bounded. Then we make a change of variables in the $s$ integration so that

$$
\xi_{4} P(s, t, u)=\sum e_{j}(\xi) s^{a_{j}} t^{b_{j}} u^{d_{j}}
$$

with $\left|e_{j_{0}}(\xi)\right|=1$ for some $j_{0}$ and $\left|e_{j}(\xi)\right| \leq 1$ for all $j$. The range of $s$ integration is no longer contained in $\{|s| \leq 1\}$, but only in some $\{|s| \leq B(\xi)\}$. However the contribution from $1 \leq|s| \leq B(\xi)$ is at most $C /|t|^{\delta}|u|^{\rho}$ for some positive $\delta$ and $\rho$. Since the $s$ integral is also uniformly bounded, we can take $\rho$ and $\delta$ to be arbitrarily small. This gives a bounded contribution in the range where $|s| \geq 1$. In the range of integration where $|s| \leq 1$, we do the $t$ integral first. Clearly for $\delta$ sufficiently small (since all $\left|e_{j}\right| \leq 1$ ), the contribution from $|t| \leq 1 /|s|^{\delta}$ gives a bounded integral. For the portion of the integral with $|t| \geq 1 /|s|^{\delta}$, we apply the estimate for $J$ in Lemma 2.4.

The estimate for $J_{5}$ is the same as the estimate $J_{4}$. In fact the first step in the estimate for $J_{4}$ was a reduction to an integral of the type $J_{5}$. 


\section{Reduction to the skeleton of the Newton polyhedron}

The purpose of this section is to show that the monomials $s^{a_{j}} t^{b_{j}} u^{d_{j}}$ arising in a polynomial $P$ for which $\left(a_{j}, b_{j}, d_{j}\right)$ lie in the interior of the Newton polyhedron of $P$ or in the interior of a face of the Newton polyhedron of $P$ play no role in the $L^{2}$ boundedness of $H_{\mathcal{R}}^{P}$. We remind the reader that we are assuming that no vertex of the Newton polyhedron of $P$ lies in a coordinate plane.

We begin with a lemma.

Lemma 4.1. For each triple $(p, q, r)$ of non-negative integers, there exists a vertex $V=\left(v_{1}, v_{2}, v_{3}\right)$ of the Newton polyhedron of $P$ such that

$$
v_{1} p+v_{2} q+v_{3} r \leq u_{1} p+u_{2} q+u_{3} r
$$

for all $u_{1}, u_{2}, u_{3}$ in the Newton polyhedron of $P$.

Proof. Consider the function

$$
F(x, y, z)=x p+y q+z r
$$

on the closed Newton polyhedron of $P$. Since all partial derivatives of $F$ are non-negative, $F$ assumes an absolute minimum on some closed face of the Newton polyhedron. Then $F$ restricted to a face assumes an absolute minimum on an edge. Finally $F$ restricted to an edge is monotonic, and hence $F$ assumes its minimum at a vertex.

In the remainder of this section $\Delta$ will be as in Section 3 , and will again change from place to place.

Let $M_{j}=e_{j} s^{a_{j}} t^{b_{j}} u^{d_{j}}, 1 \leq j \leq K$ denote the monomials of $P$ with $e_{j} \neq 0$. Assume $\left(a_{K}, b_{K}, d_{K}\right)$ is in the interior of the Newton polyhedron of $P$ or in the interior of a face of the Newton polyhedron of $P$. Let

$$
\mathcal{R}(p, q, r)=\left\{(s, t, u): 2^{-p} \leq|s| \leq 2^{-p+1}, 2^{-q} \leq|t| \leq 2^{-q+1}, 2^{-r} \leq|u| \leq 2^{-r+1}\right\} .
$$

We then set

$$
I(p, q, r)=\int_{\mathcal{R}(p, q, r) \cap \Delta} \exp i\left\{\xi_{1} s+\xi_{2} t+\xi_{3} u+\xi_{4} \sum_{j=1}^{K} e_{j} s^{a_{j}} t^{b_{j}} u^{d_{j}}\right\} \frac{d s d t d u}{s t u},
$$

and

$$
J(p, q, r)=\int_{\mathcal{R}(p, q, r) \cap \Delta} \exp i\left\{\xi_{1} s+\xi_{2} t+\xi_{3} u+\xi_{4} \sum_{j=1}^{K-1} e_{j} s^{a_{j}} t^{b_{j}} u^{d_{j}}\right\} \frac{d s d t d u}{s t u}
$$

( $\Delta$ is the same in the integrals for $I$ and $J$. ) We then have the following estimate: 
Lemma 4.2. Suppose the Newton polyhedron of P satisfies hypotheses (H1) and (H3). Then

$$
\sum_{\substack{p \geq 0 \\ q \geq 0 \\ r \geq 0}}|I(p, q, r)-J(p, q, r)| \leq C .
$$

Proof. Let $\mathcal{U}=\{(s, t, u): 1 \leq|s| \leq 2,1 \leq|t| \leq 2,1 \leq|u| \leq 2\}$. By changing variables, we see

$$
\begin{aligned}
I(p, q, r)=\int_{\mathcal{U} \cap \Delta} \exp ( & i \xi_{1} 2^{-p} s+i \xi_{2} 2^{-q} t+i \xi_{3} 2^{-r} u \\
& \left.+i \xi_{4} \sum_{j=1}^{K} e_{j} s^{a_{j}} t^{b_{j}} u^{d_{j}} 2^{-a_{j} p} 2^{-b_{j} q} 2^{-d_{j} r}\right) \frac{d s d t d u}{s t u}
\end{aligned}
$$

and

$$
\begin{aligned}
J(p, q, r)=\int_{\mathcal{U} \cap \Delta} \exp ( & i \xi_{1} 2^{-p} s+i \xi_{2} 2^{-q} t+i \xi_{3} 2^{-r} u \\
& \left.+i \xi_{4} \sum_{j=1}^{K-1} e_{j} s^{a_{j}} t^{b_{j}} u^{d_{j}} 2^{-a_{j} p} 2^{-b_{j} q_{2}} 2^{-d_{j} r}\right) \frac{d s d t d u}{s t u} .
\end{aligned}
$$

For each vertex $V_{0}=\left(a_{j_{0}}, b_{j_{0}}, d_{j_{0}}\right)$, set

$$
Z\left(V_{0}\right)=\left\{(p, q, r): V_{0} \cdot(p, q, r) \leq W_{j} \cdot(p, q, r) \text { for all } j, 1 \leq j \leq K\right\}
$$

where $W_{j}=\left(a_{j}, b_{j}, d_{j}\right)$. Note that $W_{j_{0}}=V_{0}$.

By Lemma 4.1 each $(p, q, r)$ belongs to some $Z\left(V_{0}\right)$. For a fixed $V_{0}$ suppose $N$ edges, $E_{j}$, of the Newton polyhedron emanate from $V_{0}$. Let $B_{1}, \ldots, B_{N}$ be integer lattice points on the edges $E_{j}$ emanating from $V_{0}$, one on each edge, with each $B_{j}$ different from $V_{0}$. Then for each $j, 1 \leq j \leq N$ and $(p, q, r)$ in $Z\left(V_{0}\right),(p, q, r) \cdot\left(B_{j}-V_{0}\right) \geq 0$. If $B_{j}$ is on a bounded edge, this follows from the definition of $Z\left(V_{0}\right)$ since $x \mapsto(p, q, r) \cdot\left(x-V_{0}\right)$ restricted to an edge is monotone. If $B_{j}$ is on an infinite edge, this follows because all of the coordinates $B_{j}-V_{0}$ are non-negative. Order the edges $E_{j}$ emanating from $V_{0}$ so that the pairs $\left\{E_{j}, E_{j+1}\right\},(1 \leq j \leq N-1)$ and $\left\{E_{1}, E_{N}\right\}$ span all the faces containing $V_{0}$. For $1 \leq j \leq N-1$, let

$$
F_{j}=\left\{y \in \mathbb{R}^{3}: y=\lambda_{1}\left(B_{j}-V_{0}\right)+\lambda_{2}\left(B_{j+1}-V_{0}\right) \text { with } \lambda_{1}>0 \text { and } \lambda_{2}>0\right\}
$$

and set

$$
F_{N}=\left\{y \in \mathbb{R}^{3}: y=\lambda_{1}\left(B_{N}-V_{0}\right)+\lambda_{2}\left(B_{1}-V_{0}\right) \text { with } \lambda_{1}>0 \text { and } \lambda_{2}>0\right\} .
$$


We claim that there is a point $W_{K}^{\prime}$ such that the coordinates of $W_{K}-W_{K}^{\prime}$ are non-negative and $W_{K}^{\prime}$ lies in some $F_{j}, 1 \leq j \leq N$. To see this, we may assume $W_{K}$ is not in one of the $F_{j}$ (for otherwise we could take $W_{K}^{\prime}=W_{K}$.) If $W_{K}=\left(a_{K}, b_{K}, d_{K}\right)$, let $W_{K}^{\prime \prime}=\left(a_{K}, b_{K}, f_{K}\right)$ where $f_{K}<d_{K}$ and $W_{K}^{\prime \prime}$ is in the closure of some $F_{j}$. If $W_{K}^{\prime \prime}$ is in $F_{j}$ we can take $W_{K}^{\prime}=W_{K}^{\prime \prime}$. Otherwise, $W_{K}^{\prime \prime}$ lies on some edge of $F_{j}$, and then we can take $W_{K}^{\prime}=\left(a_{K}-\delta_{1}, b_{K}-\right.$ $\left.\delta_{2}, f_{K} \pm \delta_{3}\right)$ for some small $\delta_{1}, \delta_{2}$ and $\delta_{3}$. For simplicity of notation assume $W_{K}^{\prime} \in F_{1}$.

Then

$$
\begin{aligned}
(p, q, r) \cdot\left(W_{K}-V_{0}\right) & \geq(p, q, r) \cdot\left(W_{K}^{\prime}-V_{0}\right) \\
& =\lambda_{1}(p, q, r) \cdot\left(B_{1}-V_{0}\right)+\lambda_{2}(p, q, r) \cdot\left(B_{2}-V_{0}\right)
\end{aligned}
$$

for some $\lambda_{1}>0, \lambda_{2}>0$.

Now $(p, q, r) \cdot\left(B_{1}-V_{0}\right) \geq 0$ and $(p, q, r) \cdot\left(B_{2}-V_{0}\right) \geq 0$ for $(p, q, r) \in Z\left(V_{0}\right)$. Also $(p, q, r) \cdot\left(B_{j}-V_{0}\right)$ are integers. Now we have two estimates

$$
|I(p, q, r)-J(p, q, r)| \leq A\left(\left|\xi_{4}\right| 2^{-p a_{K}} 2^{-q b_{K}} 2^{-r d_{K}}\right)^{\rho}
$$

and

$$
|I(p, q, r)-J(p, q, r)| \leq A\left(\left|\xi_{4}\right| 2^{-p a_{j_{0}}} 2^{-q b_{j_{0}}} 2^{-r d_{j_{0}}}\right)^{-\rho}
$$

for some $\rho>0$.

Let

$$
S_{L}=\left\{(p, q, r):(p, q, r) \cdot\left(B_{1}-V_{0}\right)=L\right\}
$$

and

$$
T_{M}=\left\{(p, q, r):(p, q, r) \cdot\left(B_{2}-V_{0}\right)=M\right\} .
$$

For $(p, q, r) \in S_{L} \cap T_{M}$, (4.1) becomes

$$
|I(p, q, r)-J(p, q, r)| \leq A\left(\left|\xi_{4}\right| 2^{-p a_{j_{0}}} 2^{-q b_{j_{0}}} 2^{-r d_{j_{0}}}\right)^{\rho} 2^{-\lambda_{1} \rho L} 2^{-\lambda_{2} \rho M} .
$$

Now $B_{2}-V_{0}$ and $B_{1}-V_{0}$ are not parallel. Thus if $(p, q, r)$ is in $S_{L} \cap T_{M}$, $(p, q, r)$ is on a line - namely the intersection of the planes

$$
\left\{x: x \cdot\left(B_{1}-V_{0}\right)=L\right\} \quad \text { and } \quad\left\{x: x \cdot\left(B_{2}-V_{0}\right)=M\right\} .
$$

Thus for $\left(p_{j}, q_{j}, r_{j}\right) \in S_{L} \cap T_{M}$, we can write

$$
\begin{aligned}
p_{j} & =G_{1} t_{j}+H_{1}(L, M) \\
q_{j} & =G_{2} t_{j}+H_{2}(L, M) \\
r_{j} & =G_{3} t_{j}+H_{3}(L, M)
\end{aligned}
$$


with $\left|t_{j}-t_{j+1}\right|$ bounded below and not all of $G_{1}, G_{2}, G_{3}$ zero. Now hypothesis (H3) guarantees that $\left(G_{1}, G_{2}, G_{3}\right)$ is not perpendicular to $V_{0}$, and so from (4.2) and (4.3) we see that

$$
\sum_{(p, q, r) \in S_{L} \cap T_{M}}|I(p, q, r)-J(p, q, r)| \leq A 2^{-\eta L} 2^{-\eta M}
$$

for some $\eta>0$. Then summing over $L$ and $M$, we find

$$
\sum_{(p, q, r) \in Z\left(V_{0}\right)}|I(p, q, r)-J(p, q, r)| \leq A
$$

Finally summing over all $V_{0}$ gives Lemma 4.2 .

Now by repeated use of Lemma 4.2 we arrive at the following conclusion:

Lemma 4.3. Let $P=\sum_{j} e_{j} s^{a_{j}} t^{b_{j}} u^{d_{j}}$ and $P^{\prime}=\sum_{j}^{\prime} e_{\ell} s^{a_{j}} t^{b_{j}} u^{d_{j}}$ where $\sum^{\prime}$ denotes summation over those $j$ for which $\left(a_{j}, b_{j}, d_{j}\right)$ lies on some edge of the Newton polyhedron of $P$. Suppose the Newton polyhedron of $P$ satisfies hypotheses $(H 1)$ and $(H 3)$. Then the operator $H_{\mathcal{R}}^{P}$ is bounded on $L^{2}\left(\mathbb{R}^{4}\right)$ if and only if $H_{\mathcal{R}}^{P^{\prime}}$ is bounded on $L^{2}\left(\mathbb{R}^{4}\right)$.

\section{Reduction to edge operators}

Let $E$ be a bounded edge of the Newton polyhedron of $P$ with $V_{1}, V_{2}, \ldots, V_{N-1}$, $V_{N}(N \geq 2)$ the points on $E$ corresponding to monomials of $P$ numbered so that $V_{1}$ and $V_{N}$ are vertices of the polyhedron, $V_{2}$ is closest to $V_{1}$ and $V_{N-1}$ is closest to $V_{N}$. Suppose that $V_{j}$ corresponds to the monomial $e_{j} s^{a_{j}} t^{b_{j}} u^{d_{j}}$. We then set $Q_{E}=\sum e_{j} s^{a_{j}} t^{b_{j}} u^{d_{j}}$.

To each $E$ we shall associate a region $\mathcal{R}(E)$ and an operator $H_{\mathcal{R}(E)}^{Q_{E}}$

$$
H_{\mathcal{R}(E)}^{Q_{E}} f\left(x_{1}, x_{2}, x_{3}, x_{4}\right)=\iiint_{\mathcal{R}(E)} f\left(x_{1}-s, x_{2}-t, x_{3}-u, x_{4}-Q_{E}(s, t, u)\right) \frac{d s d t d u}{s t u} .
$$

The object of this section is to show that if each $H_{\mathcal{R}(E)}^{Q_{E}}$ is bounded, then $H$ is bounded. We shall be assuming hypotheses (H1), (H2) and (H3) on the Newton polyhedron of $P$ throughout this section.

In defining $\mathcal{R}(E)$ for an edge $E$ we let $e_{\ell} s^{a_{\ell}} t^{b_{\ell}} u^{d_{\ell}}$ run through all the monomials of $P$. Again $e_{j} s^{a_{j}} t^{b_{j}} u^{d_{j}}, 1 \leq j \leq N$, are the monomials in $P$ such that $\left(a_{j}, b_{j}, d_{j}\right)$ lie on an edge $E$ ordered as above. 
We let

$$
\begin{aligned}
\mathcal{R}_{1}(E)=\{ & (s, t, u):\left|s^{a_{1}} t^{b_{1}} u^{d_{1}}\right| \geq\left|s^{a_{2}} t^{b_{2}} u^{d_{2}}\right| \text { and }\left|s^{a_{2}} t^{b_{2}} u^{d_{2}}\right| \geq\left|s^{a_{\ell}} t^{b_{\ell}} u^{d_{\ell}}\right| \\
& \text { for all }\left(a_{\ell}, b_{\ell}, d_{\ell}\right) \text { corresponding to monomials of } P \text { other } \\
& \text { than } \left.\left(a_{1}, b_{1}, d_{1}\right)\right\}
\end{aligned}
$$

and

$$
\begin{aligned}
\mathcal{R}_{2}(E)=\{ & (s, t, u):\left|s^{a_{N}} t^{b_{N}} u^{d_{N}}\right| \geq\left|s^{a_{N-1}} t^{b_{N-1}} u^{d_{N-1}}\right| \text { and }\left|s^{a_{N-1}} t^{b_{N-1}} u^{d_{N-1}}\right| \\
& \geq\left|s^{a_{\ell}} t^{b_{\ell}} u^{d_{\ell}}\right| \text { for all }\left(a_{\ell}, b_{\ell}, d_{\ell}\right) \text { corresponding to monomials of } P \\
& \text { other than } \left.\left(a_{N}, b_{N}, d_{N}\right)\right\} .
\end{aligned}
$$

Then put

$$
\mathcal{R}(E)=\left(\mathcal{R}_{1}(E) \cup \mathcal{R}_{2}(E)\right) \cap\{(s, t, u):|s|,|t|,|u| \leq 1\} .
$$

Lemma 5.1. Suppose each monomial of $P$ corresponds to a point on an edge of the Newton polyhedron of $P$. Moreover assume each infinite edge contains only one point corresponding to a monomial of $P$. Then each $(s, t, u)$ with $|s|,|t|,|u| \leq 1$ is in some $\mathcal{R}(E)$ and the $\mathcal{R}(E)$ have disjoint interiors.

Proof. For each $(s, t, u)$ there is a vertex $V_{1}=\left(a_{1}, b_{1}, d_{1}\right)$ such that $\left|s^{a_{1}} t^{b_{1}} u^{d_{1}}\right|$ $\geq\left|s^{a_{\ell}} t^{b_{\ell}} u^{d_{\ell}}\right|$ for all $\left(a_{\ell}, b_{\ell}, d_{\ell}\right)$. (The proof of this is the same as that of Lemma 4.1.) Let $L_{1}, \ldots, L_{K}$ be the bounded edges emanating from $V_{1}$. On each $L_{\ell}$ let $U_{\ell}=\left(a_{j_{\ell}}, b_{j_{\ell}}, d_{j_{\ell}}\right)$ be the point closest to $V_{1}$. Choose $E$ to be that $L_{\ell}$ such that $\left|s^{a_{j_{\ell}}} t^{b_{j_{\ell}}} u^{d_{j_{\ell}}}\right|$ is maximal for $1 \leq \ell \leq K$. Let us say $\ell=1$.

Change notation so that $U_{1}=\left(a_{2}, b_{2}, d_{2}\right)$ and introduce $\sigma=\log (1 / s)$, $\tau=\log (1 / t)$ and $v=\log (1 / u)$. Observe that

$$
(x, y, z) \mapsto x \sigma+y \tau+z v
$$

is monotone when restricted to each edge. Thus if $W=\left(a_{\ell}, b_{\ell}, d_{\ell}\right)$ is a point on any $L_{\ell}, 1 \leq \ell \leq K$, then

$$
\left|s^{a_{1}} t^{b_{1}} u^{d_{1}}\right| \geq\left|s^{a_{2}} t^{b_{2}} u^{d_{2}}\right| \geq\left|s^{a_{\ell}} t^{b_{\ell}} u^{d_{\ell}}\right| .
$$

Now suppose $W$ is not on one of the edges emanating from $V_{1}$. Let $R_{k}$ denote points on the infinite edges, one $R_{k}$ on each infinite edge emanating from $V_{1}$. Then since $W$ is on some bounded edge not emanating from $V_{1}$,

$$
W-V_{1}=\sum_{\ell=1}^{K} \lambda_{\ell}\left(U_{\ell}-V_{1}\right)+\sum_{k} \eta_{k}\left(R_{k}-V_{1}\right)
$$


where each $\eta_{k} \geq 0$, and $\Sigma \lambda_{\ell}+\Sigma \eta_{k} \geq 1-$ for if $\Sigma \lambda_{\ell}+\Sigma \eta_{k}<1, W$ would be in the interior of the Newton polyhedron or in the interior of a face of the Newton polyhedron or on an edge emanating from $V_{1}$.

It is at this stage that we are using strongly the reduction to the 1skeleton of the Newton polyhedron of $P$ in Section 4 .

Next we let the $R_{k}$ tend to infinity in such a way that the $\lambda_{\ell}$ do not change and we see then that the $\eta_{k}$ must tend to 0 . Therefore $\sum_{\ell=1}^{K} \lambda_{\ell} \geq 1$, and so

$$
\begin{aligned}
\left(a_{\ell}-a_{1}\right) \sigma+\left(b_{\ell}-b_{1}\right) \tau & +\left(d_{\ell}-d_{1}\right) v \\
& \geq \sum_{\ell=1}^{K} \lambda_{\ell}\left[\left(a_{j_{\ell}}-a_{1}\right) \sigma+\left(b_{j_{\ell}}-b_{1}\right) \tau+\left(d_{j_{\ell}}-d_{1}\right) v\right] \\
& \geq\left(\sum_{\ell=1}^{K} \lambda_{\ell}\right)\left[\left(a_{2}-a_{1}\right) \sigma+\left(b_{2}-b_{1}\right) \tau+\left(d_{2}-d_{1}\right) v\right] \\
& \geq\left[\left(a_{2}-a_{1}\right) \sigma+\left(b_{2}-b_{1}\right) \tau+\left(d_{2}-d_{1}\right) v\right] .
\end{aligned}
$$

Thus since $|s| \leq 1,|t| \leq 1$, and $|u| \leq 1$

$$
\left|s^{a_{\ell}} t^{b_{\ell}} u^{d_{\ell}}\right| \leq\left|s^{a_{2}} t^{b_{2}} u^{d_{2}}\right|,
$$

and $(s, t, u)$ is in $\mathcal{R}_{1}(E)$. It is clear that the $\mathcal{R}(E)$ are disjoint and Lemma 5.1 is proved.

Now let $E$ be an edge, and let

$$
P(s, t, u)=Q_{E}(s, t, u)+R(s, t, u)+e_{L+1} s^{a_{L+1}} t^{b_{L+1}} u^{d_{L+1}}
$$

where

$$
Q_{E}(s, t, u)=\sum_{j=1}^{L} e_{j} s^{a_{j}} t^{b_{j}} u^{d_{j}},
$$

with $\left(a_{j}, b_{j}, d_{j}\right)$ on $E$ for $1 \leq j \leq L$, and where $R$ and $e_{L+1} s^{a_{L+1}} t^{b_{L+1}} u^{d_{L+1}}$ corresponds to monomials of $P$ on the 1-sketeton but not on the edge $E$.

Then we have:

Lemma 5.2. Let

$$
I\left(\xi_{1}, \xi_{2}, \xi_{3}, \xi_{4}\right)=\int_{\mathcal{R}(E)} e^{i \xi_{1} s} e^{i \xi_{2} t} e^{i \xi_{3} u} e^{i \xi_{4} P(s, t, u)} \frac{d s d t d u}{s t u}
$$

and

$$
J\left(\xi_{1}, \xi_{2}, \xi_{3}, \xi_{4}\right)=\int_{\mathcal{R}(E)} e^{i \xi_{1} s} e^{i \xi_{2} t} e^{i \xi_{3} u} e^{i \xi_{4}\left(Q_{E}+R\right)(s, t, u)} \frac{d s d t d u}{s t u} .
$$

Then $I(\xi)-J(\xi)$ is uniformly bounded. 
Proof. It suffices to consider the integral over $\mathcal{R}_{1}(E)$. Let us suppose $V_{1}=\left(a_{1}, b_{1}, d_{1}\right)$ is a vertex of $E$ and $V_{2}=\left(a_{2}, b_{2}, d_{2}\right)$ is the point on $E$ closest to $V$. For $p \geq 0, q \geq 0, r \geq 0$, set

$$
A(p, q, r)=\left\{(s, t, u): 2^{-p} \leq|s| \leq 2^{-p+1}, 2^{-q} \leq|t| \leq 2^{-q+1}, 2^{-r} \leq|u| \leq 2^{-r+1}\right\} .
$$

Put

$$
I(p, q, r)=\int_{\mathcal{R}_{1}(E) \cap A(p, q, r)} \exp \left(i \xi_{1} s+i \xi_{2} t+i \xi_{3} u+i \xi_{4} P(s, t, u)\right) \frac{d s d t d u}{s t u},
$$

and

$$
J(p, q, r)=\int_{\mathcal{R}_{1}(E) \cap A(p, q, r)} \exp \left(i \xi_{1} s+i \xi_{2} t+i \xi_{3} u+i \xi_{4}\left(Q_{E}+R\right)(s, t, u)\right) \frac{d s d t d u}{s t u} .
$$

Arguing as in Lemma 4.2 we then have the following estimates:

$$
|I(p, q, r)-J(p, q, r)| \leq A\left(\left|\xi_{4}\right| 2^{-p a_{L+1}} 2^{-q b_{L+1}} 2^{-r d_{L+1}}\right)^{\eta}
$$

and

$$
|I(p, q, r)-J(p, q, r)| \leq A\left(\left|\xi_{4}\right| 2^{-p a_{1}} 2^{-q b_{1}} 2^{-r d_{1}}\right)^{-\eta}
$$

for some $\eta>0$.

We want to see that we can replace the estimate (5.1) by

$$
|I(p, q, r)-J(p, q, r)| \leq A\left(\left|\xi_{4}\right| 2^{-p \alpha} 2^{-q \beta} 2^{-r \delta}\right)^{\eta}
$$

where the point $W=(\alpha, \beta, \delta)$ does not lie on $E$, and the plane determined by $W, V_{1}$ and $V_{2}$ does not contain the origin. If $\left(a_{L+1}, b_{L+1}, d_{L+1}\right)$ lies on an edge meeting $E$, we may take $W=\left(a_{L+1}, b_{L+1}, d_{L+1}\right)$. For then if the plane determined by $V_{1}, V_{2}$ and $\left(a_{L+1}, b_{L+1}, d_{L+1}\right)$ contained the origin, the plane determined $V_{1},\left(a_{L}, b_{L}, d_{L}\right)$ and the other vertex of the edge containing $\left(a_{L+1}, b_{L+1}, d_{L+1}\right)$ would determine a plane containing the origin contradicting assumption (H3). (The edge containing $\left(a_{L+1}, b_{L+1}, d_{L+1}\right)$ could not be infinite since we are assuming each infinite edge contains only one point.) If $\left(a_{L+1}, b_{L+1}, d_{L+1}\right)$ lies on some edge not meeting $E$, we may take $W$ to be one vertex on that edge since $p x+q y+r z$ is monotone along edges.

If $\mathcal{R}_{1}(E) \cap A(p, q, r) \neq \emptyset$, then $(p, q, r) \cdot\left(V_{2}-V_{1}\right) \geq-B$ and $(p, q, r)$. $\left(W-V_{2}\right) \geq-B$ for some positive $B$. Let

$$
F_{N}=\left\{(p, q, r):\left(V_{2}-V_{1}\right) \cdot(p, q, r)=N\right\}
$$

and

$$
D_{M}=\left\{(p, q, r):\left(W-V_{2}\right) \cdot(p, q, r)=M\right\}
$$


Now $I(\xi)-J(\xi)$ is the sum of two terms, the first of which is dominated by $\sum_{p, q, r \geq 0}|I(p, q, r)-J(p, q, r)|=\sum_{N \geq-B} \sum_{-B \leq M \leq N+B} \sum_{(p, q, r) \in F_{N} \cap D_{M}}|I(p, q, r)-J(p, q, r)|$.

We may rewrite (5.3) as

$$
|I(p, q, r)-J(p, q, r)| \leq A 2^{-\eta N}\left(\left|\xi_{4}\right| 2^{-\left(a_{1} p+b_{1} q+d_{1} r\right)}\right)^{\eta}
$$

Since $V_{1}, V_{2}$ and $W$ are not collinear, $F_{N} \cap D_{M}$ is a line. Arguing once more as in Lemma 4.2 we can sum now in $p, q$, and $r$ as long as the line of intersection of the planes $F_{N}$ and $D_{M}$ is not perpendicular to $\left(a_{1}, b_{1}, d_{1}\right)$. And this condition is satisfied since the plane through $V_{1}, V_{2}$ and $W$, which is the plane determined by $E$ and $W$, does not contain the origin.

By applying Lemma 4.3, Lemma 5.1 and Lemma 5.2 many times we obtain the following conclusion:

Proposition 5.3. Suppose the Newton polyhedron of $P$ satisfes hypotheses (H1), (H2) and (H3). If $H_{\mathcal{R}(E)}^{Q_{E}}$ is bounded on $L^{2}\left(\mathbb{R}^{4}\right)$ for each bounded edge $E$, then $H_{\mathcal{R}}^{P}$ is bounded on $L^{2}\left(\mathbb{R}^{4}\right)$.

\section{A further reduction for edge operators}

In this section $\Delta$ is as in Section 3. Suppose that

$$
Q(s, t, u)=\sum_{j=1}^{L} e_{j} s^{a_{j}} t^{b_{j}} u^{d_{j}}
$$

where $\left(a_{j}, b_{j}, d_{j}\right)$ lie on some line. We assume the hypotheses (H1) and (H2), that is each of $a_{j}, b_{j}$ and $d_{j}$ is positive, each of the sequences $\left\{a_{j}\right\},\left\{b_{j}\right\}$ and $\left\{d_{j}\right\}$ is strictly monotonic and that the projection of the line through the $\left(a_{j}, b_{j}, d_{j}\right)$ onto a coordinate plane does not pass through the origin. Furthermore we shall also assume that at least two of $a_{1}, b_{1}$ and $d_{1}$ are even, at least two of $a_{L}, b_{L}, d_{L}$ are even.

Proposition 6.1. Let $\mathcal{R}=\{(s, t, u):|s| \leq 1,|t| \leq 1,|u| \leq 1\}$ or let $\mathcal{R}=\mathbb{R}^{3}$. Then

$$
\begin{aligned}
m(\xi) & :=\int_{\mathcal{R} \cap \Delta} e^{i \xi_{1} s} e^{i \xi_{2} t} e^{i \xi_{3} u} e^{i \xi_{4} Q(s, t, u)} \frac{d s d t d u}{s t u} \\
& =\int_{\mathcal{R}_{\xi} \cap \Delta} e^{i \xi_{4} Q(s, t, u)} \frac{d s d t d u}{s t u}+O(1)
\end{aligned}
$$

where

$$
\mathcal{R}_{\xi}=\left\{(s, t, u):|s| \leq \frac{1}{\left|\xi_{1}\right|},|t| \leq \frac{1}{\left|\xi_{2}\right|},|u| \leq \frac{1}{\left|\xi_{3}\right|}\right\}
$$


Remark. Proposition 6.1 together with Proposition 5.3 implies the sufficiency result in Theorem 1.1. The point is that in this case the hypothesis of Theorem 1.1 implies

$$
\int_{\mathcal{R}_{\xi} \cap \Delta} e^{i \xi_{4} Q(s, t, u)} \frac{d s d t d u}{s t u}=0 .
$$

By applying the lemmas of Section 3, we see that except for a bounded error $m(\xi)$ is a sum of an integral $I(\xi)$ and three integrals of the type $J(\xi)$, where

$$
I(\xi)=\int_{\mathcal{R}_{4} \cap \Delta} e^{i \xi_{1} s} e^{i \xi_{2} t} e^{i \xi_{3} u} s^{i \xi_{4} Q(s, t, u)} \frac{d s d t d u}{s t u}
$$

and

$$
J(\xi)=\int_{\mathcal{R}_{3} \cap \Delta} e^{i \xi_{2} u} e^{i \xi_{4} Q(s, t, u)} \frac{d s d t d u}{s t u},
$$

where

$$
\mathcal{R}_{3}=\left\{(s, t, u):|s| \leq \frac{1}{\left|\xi_{1}\right|},|t| \geq \frac{1}{\left|\xi_{2}\right|},|u| \leq \frac{1}{\left|\xi_{3}\right|}\right\}
$$

and

$$
\mathcal{R}_{4}=\left\{(s, t, u):|s| \leq \frac{1}{\left|\xi_{1}\right|},|t| \leq \frac{1}{\left|\xi_{2}\right|}, \quad|u| \leq \frac{1}{\left|\xi_{3}\right|}\right\} .
$$

We begin with $I(\xi)$.

Lemma 6.2. Assume $b_{1}$ and $b_{L}$ are even. Then

$$
\int_{\mathcal{R}_{4} \cap \Delta}\left(e^{i \xi_{1} s} e^{i \xi_{3} u}-1\right) e^{i \xi_{2} t} e^{i \xi_{4} P(s, t, u)} \frac{d s d t d u}{s t u}=O(1) .
$$

Remark. Lemma 6.2 does not use the hypothesis on the parity of $a_{1}, a_{L}, d_{1}$ or $d_{L}$.

Proof. Write $e^{i \xi_{1} s} e^{i \xi_{3} u}-1=\left(e^{i \xi_{1} s}-1\right)\left(e^{i \xi_{3} u}-1\right)+\left(e^{i \xi_{3} u}-1\right)+\left(e^{i \xi_{1} s}-1\right)$

The contribution from $\left(e^{i \xi_{1} s}-1\right)\left(e^{i \xi_{3} u}-1\right)$ is bounded because the $t$ integral is uniformly bounded. The contributions from $e^{i \xi_{3} u}-1$ and $e^{i \xi_{1} s}-1$ are treated in a similar manner, so it suffices to deal with $I_{1}$ where

$$
I_{1}=\int_{\mathcal{R}_{4} \cap \Delta}\left(e^{i \xi_{1} s}-1\right) e^{i \xi_{2} t} e^{i \xi_{4} Q(s, t, u)} \frac{d s d t d u}{s t u} .
$$

By doing the $u$ integration first, we see we can replace $e^{i \xi_{2} t}$ by 1 . Thus we have to consider

$$
I_{2}=\int_{\mathcal{R}_{4} \cap \Delta} \frac{e^{i \xi_{1} s}-1}{s} e^{i \xi_{4} Q(s, t, u)} d s \frac{d t d u}{t u} .
$$


We shall make a change of variables. First we divide the integral into four regions depending on the sign of $s$ and $u$. For $s$ and $u$ positive we make the change of variables $t=t^{\prime} s^{\alpha} u^{\beta}$ with $\alpha=\frac{a_{2}-a_{1}}{b_{1}-b_{2}}$ and $\beta=\frac{d_{2}-d_{1}}{b_{1}-b_{2}}$. We find

$$
I_{2}=\int_{\mathcal{R}_{5} \cap \Delta} \frac{e^{i \xi_{1} s}-1}{s} e^{i \xi_{4} s^{\rho_{1}} u^{\rho_{2}} Q(1, t, 1)} d s \frac{d t d u}{t u}
$$

where

$$
\rho_{1}=\frac{b_{1} a_{2}-a_{1} b_{2}}{b_{2}-b_{1}} \quad \text { and } \quad \rho_{2}=\frac{b_{1} d_{2}-d_{1} b_{2}}{b_{2}-b_{1}},
$$

and $\rho_{1}$ and $\rho_{2}$ are not zero because of the hypothesis that the projection of the line through $\left(a_{j}, b_{j}, d_{j}\right)$ does not pass through the origin. $\mathcal{R}_{5}$ is contained in $\left\{|s| \leq \frac{1}{\left|\xi_{1}\right|}\right\}$ and the $t$ integration is over a finite union of intervals symmetric around 0 . Now by making a change of variables first in the $s$ integral and then in the $u$ integral we may assume $\xi_{1}=1$ and $\xi_{4}=1$. Since $b_{1}$ and $b_{N}$ are even, we may apply Lemma 2.4 to the $t$ integral to obtain the estimates $A /\left(s^{\rho_{1}} u^{\rho_{2}}\right)^{\eta}$ and $A\left(s^{\rho_{1}} u^{\rho_{2}}\right)^{\eta}$ for some $\eta>0$. Since the $t$ integral is bounded we obtain the estimates $A / s^{\delta} u^{\delta}$ and $A u^{\delta} / s^{\delta}$ for some small $\delta<1 / 2$. We apply the first estimate to the region $u \geq 1$ and the second when $u \leq 1$. Since $0 \leq s \leq 1$, the lemma is proved.

\section{Lemma 6.3.}

$$
\int_{\mathcal{R}_{4} \cap \Delta} \frac{e^{i \xi_{2} t}-1}{t} e^{i \xi_{4} Q(s, t, u)} \frac{d s d t d u}{s u}
$$

is bounded.

Proof. Consider $t>0$. In the $s, u$ integral we can make a change of variable $s=t^{\alpha} \sigma, u=t^{\beta} v$ so that $Q(s, t, u)$ becomes $Q(\sigma, 1, v)$. This requires $\alpha$ and $\beta$ to satisfy the equations

$$
b_{j}-b_{1}+\alpha\left(a_{j}-a_{1}\right)+\beta\left(d_{j}-d_{1}\right)=0
$$

and

$$
b_{1}+\alpha a_{1}+\beta d_{1}=0
$$

or

$$
\alpha \frac{a_{j}-a_{1}}{b_{j}-b_{1}}+\beta \frac{d_{j}-d_{1}}{b_{j}-b_{1}}=-1
$$

and

$$
\alpha a_{1}+\beta d_{1}=0 .
$$

These equations have a solution if

$$
\frac{a_{1}}{d_{1}} \neq \frac{a_{j}-a_{1}}{d_{j}-d_{1}}
$$


Thus is just the condition that the projection of the line through the $\left(a_{j}, b_{j}, d_{j}\right)$ onto the $x-z$ plane does not go through the origin. Since at least one of the $a_{1}$ and $d_{1}$ is even and at least one of $a_{L}$ and $d_{L}$ is even, Lemma 6.2 now follows from Lemma 2.6.

Lemma 6.4. $J(\xi)$ is uniformly bounded.

Proof. By changing variables in the $t$-integral, we can write

$$
J=\int_{1}^{\infty} \frac{e^{i t}}{t} \iint_{\Delta} e^{i \xi_{4} Q(s, t, u)} \frac{d s d u}{s u} d t .
$$

This change of variables of course changes the coefficients of $Q$. We can then make a further change of variables in $s$ and $u$, so that we return to the original $Q$. We want to return to the original $Q$ because we want to use Lemma 2.6 in which the estimates depend upon the coefficients of $Q$.

Then by making a further change of variables as in the proof of Lemma 6.3, we see

$$
J=\int_{1}^{\infty} \frac{e^{i t}}{t} \iint_{\Delta} e^{i \xi_{4} Q(s, 1, u)} \frac{d s d u}{s u} d t .
$$

By a yet further change of variables we may assume $\xi_{4}= \pm 1$ without changing the coefficients of $Q$. We shall take $\xi_{4}=1$.

Let us consider first the region where $|s| \leq 1$ and $|u| \leq 1$. We see the contribution for $|s u| \leq \frac{1}{t}$ is bounded by subtracting $e^{i Q(s, 1, u)}$. In the region where $|s u| \geq \frac{1}{t}$, we do the $t$ integral first. We are then integrating over a bounded number of intervals in each of which $t \geq 1 /|s u|$. Thus the contribution from the $t$ integral is at most $C|s u|$. This finishes the discussion of $\{(s, u):|s| \leq 1$, and $|u| \leq 1\}$.

Let us next consider the region where $|s| \geq|u| \geq 1$. If we integrate in $|s| \geq t$, we gain a positive power of $\frac{1}{t|u|}$, and this contribution is bounded. If $t \geq|s|$ we perform the $t$ integral first gaining $\frac{1}{|s|}$, we then have the estimate

$$
\int_{|s| \geq 1} \frac{d s}{|s|^{2}} \int_{1 \leq|u| \leq|s|} \frac{1}{|u|} d u=O(1)
$$

It remains to consider the two "corridors"

$$
\{(s, u):|s| \geq 1,|u| \leq 1\} \quad \text { and } \quad\{(s, u):|s| \leq 1,|u| \geq 1\} .
$$

We consider the region $\{(s, u):|s| \geq 1,|u| \leq 1\}$. The other region is similar.

$$
\left|\iint_{\substack{|s| \geq t,|u| \leq 1 \\ \Delta}} e^{i Q(s, 1, u)} \frac{d s d u}{s u}\right| \leq \frac{C}{t^{\epsilon}}
$$


for some $\epsilon>0$ by Lemma 2.6. Similarly

$$
\left|\int_{|s| \leq t} \int_{|u| \leq \frac{1}{t}} e^{i Q(s, 1, u)}\right| \leq \frac{C}{t^{\epsilon}} .
$$

This leaves us with

$$
\int_{1}^{\infty} e^{i t} \frac{d t}{t} \iint_{\substack{1 \leq|\leq| \leq t \\ 1 \geq|u| \geq \frac{1}{t}}} e^{i Q(s, 1, u)} \frac{d s}{s} \frac{d u}{u} .
$$

In this region we do the $t$ integral first. The range of integration in $t$ will consist of a bounded union of intervals in which $t \geq|s|$ and $t \geq \frac{1}{|u|}$. So the $t$ integral is at most $C|u|^{1 / 2} /|s|^{1 / 2}$. This completes the proof of Lemma 6.4 and of Proposition 6.1.

\section{Vertex operators}

Here we consider

$$
H_{\mathcal{R}}^{V} f\left(x_{1}, x_{2}, x_{3}, x_{4}\right)=\int_{\mathcal{R}} f\left(x_{1}-s, x_{2}-t, x_{3}-u, x_{4}-s^{a} t^{b} u^{d}\right) \frac{d s d t u}{s t u} .
$$

Here we are supposing that $\mathcal{R}$ is either all of $\mathbb{R}^{3}$ or the cube $\{|s| \leq 1,|t| \leq 1$, $|u| \leq 1\}$.

$$
\begin{aligned}
\text { So } \widehat{H_{\mathcal{R}}^{V}}(\xi)= & m_{\mathcal{R}}(\xi) \hat{f}(\xi) \text {, where } \xi=\left(\xi_{1}, \xi_{2}, \xi_{3}, \xi_{4}\right) \text {, and } \\
& m_{\mathcal{R}}(\xi)=\iiint_{\mathcal{R}} e^{i \xi_{1} s} e^{i \xi_{2} t} e^{i \xi_{3} u} e^{i \xi_{4} s^{a} t^{b} u^{d}} \frac{d s d t d u}{s t u} .
\end{aligned}
$$

Lemma 7.1. (i) If at least two of $a, b$ and $d$ are even then $m_{\mathcal{R}}(\xi)$ is uniformly bounded.

(ii) If two of $a, b$ and $d$ are odd and one is even

$$
\left|m_{\mathcal{R}}(\xi)\right| \leq C \log ^{+} \frac{\left|\xi_{4}\right|}{\left|\xi_{1}\right|^{a}\left|\xi_{2}\right|^{b}\left|\xi_{3}\right|^{d}}+O(1)
$$

and if $\xi_{1}, \xi_{2}, \xi_{3}$ and $\xi_{4}$ positive

$$
m_{\mathcal{R}}(\xi)=\frac{a b \pi^{2}}{4} \log ^{+} \frac{\xi_{4}}{\xi_{1}^{a} \xi_{2}^{b} \xi_{3}^{d}}+O(1)
$$


(iii) If all of $a, b$ and $d$ are odd

$$
\left|m_{R}(\xi)\right| \leq C\left(\log ^{+} \frac{\left|\xi_{4}\right|}{\left|\xi_{1}\right|^{a}\left|\xi_{2}\right|^{b}\left|\xi_{3}\right|^{d}}\right)^{2}+O(1)
$$

and if $\xi_{1}, \xi_{2}, \xi_{3}$ and $\xi_{4}$ are positive

$$
m_{R}(\xi)=\frac{a b d \pi}{2}\left(\log ^{+} \frac{\xi_{4}}{\xi_{1}^{a} \xi_{2}^{b} \xi_{3}^{d}}\right)^{2}+O\left(\log ^{+} \frac{\xi_{4}}{\xi_{1}^{a} \xi_{2}^{b} \xi_{3}^{d}}\right)+O(1) .
$$

Proof. We consider the case $\mathcal{R}=\mathbb{R}^{3}$. The case that $\mathcal{R}$ is a bounded cube follows from a similar argument.

Part $(i)$ follows from Theorem 5.1 in [4] or alternatively from Proposition 6.1.

Let us consider part (ii). Suppose $a$ and $b$ are odd and $d$ is even. Then by Lemmas 3.1 and 6.2 matters are reduced to

$$
m_{\mathcal{R}}(\xi)=\int_{|s| \leq \frac{1}{\left|\xi_{1}\right|}} \int_{|t| \leq \frac{1}{\left|\xi_{2}\right|}} \int_{u \in \mathbb{R}} e^{i \xi_{4} s^{a} t^{b} u^{d}} e^{i \xi_{3} u} \frac{d s d t d u}{s t u}+O(1) .
$$

Let us assume $\xi_{1}, \xi_{2}, \xi_{3}$ and $\xi_{4}$ are positive. So

$$
m_{\mathcal{R}}(\xi)=\int_{|s| \leq 1} \int_{|t| \leq 1} \int_{u \in \mathbb{R}} e^{i \lambda s^{a} t^{b} u^{d}} e^{i u} \frac{d s d t d u}{s t u}+O(1) .
$$

with $\lambda=\frac{\xi_{4}}{\xi_{1}^{a} \xi_{2}^{b} \xi_{3}^{d}}$.

Thus

$$
\begin{aligned}
m_{\mathcal{R}}(\xi) & =\int_{0}^{1} \int_{0}^{1} \int_{0}^{\infty} \sin \left(\lambda s^{a} t^{b} u^{d}\right) \sin u \frac{d s d t d u}{s t u}+O(1) \\
& =a b \int_{0}^{\lambda} \frac{d s}{s} \int_{0}^{1} \frac{d t}{t} \int_{0}^{\infty} \sin \left(s t u^{d}\right) \sin u \frac{d u}{u}+O(1) \\
& =a b \int_{0}^{1}+a b \int_{1}^{\lambda}+O(1) \\
& :=a b I+a b I I+O(1) .
\end{aligned}
$$

Consider first I. The integral is

$$
\begin{aligned}
\int_{0}^{1} \frac{d s}{s} \int_{0}^{s} \frac{d v}{v} \int_{0}^{\infty} \sin u \sin \left(v u^{d}\right) \frac{d u}{u}= & \int_{0}^{1} \frac{d s}{s} \int_{0}^{s} \frac{d v}{v} \int_{0}^{\left(\frac{1}{v}\right)^{\delta}} \sin u \sin \left(v u^{d}\right) \frac{d u}{u} \\
& +\int_{0}^{1} \frac{d s}{s} \int_{0}^{s} \frac{d v}{v} \int_{\left(\frac{1}{v}\right)^{\delta}}^{\infty} \sin u \sin \left(v u^{d}\right) \frac{d u}{u} .
\end{aligned}
$$

The first integral is clearly bounded if $\delta$ is sufficiently small. The second integral is bounded in view of the second conclusion of Lemma 2.5. 
The integral in II is

$$
\int_{1}^{\lambda} \frac{d s}{s} \int_{0}^{s} \frac{d t}{t} \int_{0}^{\infty} \sin u \sin \left(t u^{d}\right) \frac{d u}{u}
$$

We want to replace the range of integration in the $t$ integral from $0 \leq t \leq s$ by $0 \leq t \leq \infty$, for if we can make this change, the integral will become $C$ $\log \lambda$, where

$$
C=\left(\int_{0}^{\infty} \frac{\sin u}{u} d u\right)^{2}=\frac{\pi^{2}}{4}
$$

We shall show that the $u$ integral is $O\left(t^{-\delta}\right)$ for some positive $\delta$ which will establish that the contribution in the range $t \geq s$ is bounded. Now

$$
\begin{aligned}
\int_{0}^{\infty} & \sin u \sin \left(t u^{d}\right) \frac{d u}{u}=\frac{1}{d t} \int_{0}^{\infty} \sin u \frac{d}{d u}\left(1-\cos \left(t u^{d}\right)\right) \frac{d u}{u^{d}} \\
& =-\frac{1}{d t} \int_{0}^{\infty}(\cos u)\left(1-\cos \left(t u^{d}\right)\right) \frac{d u}{u^{d}}+\frac{1}{t} \int_{0}^{\infty}(\sin u)\left(1-\cos \left(t u^{d}\right)\right) \frac{d u}{u^{d+1}} \\
& :=I I I+I V .
\end{aligned}
$$

For an $\eta>0$ to be chosen later,

$$
|I I I| \leq \frac{1}{t} \int_{0}^{1 / t^{\eta}} t^{2} u^{d} d u+\frac{1}{t} \int_{1 / t^{\eta}}^{\infty} \frac{d u}{u^{d}} \leq C t\left(\frac{1}{t}\right)^{(d+1) \eta}+\left(\frac{1}{t}\right) t^{\eta(d-1)}
$$

If we choose $\eta$ so that $\frac{1}{d-1}>\eta>\frac{1}{d+1}$, we see $|I I I| \leq C\left(\frac{1}{t}\right)^{\delta}$ for some $\delta>0$. The integral IV is shown to be $O\left(\left(\frac{1}{t}\right)^{\delta}\right)$ in a similar manner. This completes the discussion of part $(i i)$ of Lemma 7.1.

We turn to the third part of Lemma 7.1. Then using the results of Section 3, we see that up to a bounded error

$$
\begin{aligned}
m(\xi)=\int_{|s| \leq \frac{1}{\left|\xi_{1}\right|}} \int_{|t| \leq \frac{1}{\left|\xi_{2}\right|}} \int_{|u| \leq \frac{1}{\left|\xi_{3}\right|}} e^{i \xi_{3} u} e^{i \xi_{4} s^{a} t^{b} u^{d}} \frac{d s d t d u}{s t u} \\
+\int_{|s| \leq \frac{1}{\left|\xi_{1}\right|}} \int_{|t| \leq \frac{1}{\left|\xi_{2}\right|}} \int_{|u| \geq \frac{1}{\xi_{3} \mid}} e^{i \xi_{3} u} e^{i \xi_{4} s^{a} t^{b} u^{d}} \frac{d s d t d u}{s t u}
\end{aligned}
$$

plus two integrals similar to the last one, with the roles of $s, t$, and $u$ interchanged.

Call the two integrals $I$ and $J$. We shall first show that $J$ is $O(\log \lambda)$. By the same changes of variables,

$$
J=a b \int_{0}^{\lambda} \int_{0}^{s} \int_{1}^{\infty} \cos u \sin \left(t u^{d}\right) \frac{d s d t d u}{s t u}
$$

with $\lambda=\frac{\xi_{4}}{\xi_{1}^{a} \xi_{2}^{b} \xi_{3}^{d}}$ 
The contribution from $0 \leq s \leq 1$ is bounded. To see this first note that for a sufficiently small $\delta$ the contribution from $u \leq \frac{1}{t^{\delta}}$ is bounded. For $u \geq \frac{1}{t^{\delta}}$, we employ Corollary 3.6 of [2].

Also the $u$ integral is $O\left(1 / t^{\delta}\right)$ for large $t$, so in the $t$ integral we may replace the upper limit of integration by $\infty$ at the expense of a bounded error. So

$$
J=C \int_{1}^{\lambda} \frac{d s}{s}+O(1)=C \log \lambda+O(1)
$$

where

$$
C=a b \int_{0}^{\infty} \int_{1}^{\infty} \cos u \sin \left(t u^{d}\right) \frac{d t d u}{t u}
$$

(The reasoning above shows the integral exists.)

We turn to $I$. Note that

$$
I=\int_{|s| \leq 1} e^{i s} \frac{d s}{s} \int_{|t| \leq 1} e^{i t} \frac{d t}{t} \int_{|u| \leq 1} e^{i \lambda s^{a} t^{b} u^{d}} e^{i u} \frac{d u}{u} .
$$

We first want to show

$$
\int_{|s| \leq 1} \int_{|t| \leq 1}\left(e^{i s} e^{i t}-1\right) \frac{d t}{t} \int_{|u| \leq 1} e^{i u} e^{i \lambda s^{a} t^{b} u^{d}} \frac{d u}{u}=O(\log \lambda) .
$$

As in the proof of Lemma 6.2, it suffices to consider

$$
\begin{aligned}
\int_{|t| \leq 1} \frac{e^{i t}-1}{t} \int_{|s| \leq 1} \frac{d s}{s} \int_{|u| \leq 1} e^{i u} e^{i \lambda s^{a} t^{b} u^{d}} \frac{d u}{u} & \\
& =\int_{|t| \leq 1} \frac{e^{i t}-1}{t} \int_{0}^{\lambda^{1 / a}} \frac{d s}{s} \int_{0}^{1} \cos u \sin \left(s^{a} t^{b} u^{d}\right) \frac{d u}{u} .
\end{aligned}
$$

The contribution from $s \leq 1$ is clearly bounded. For $s \geq 1$ we can replace $\cos u$ by 1 with an error which is $O(\log \lambda)$. This leaves

$$
\int_{|t| \leq 1} \frac{e^{i t}-1}{t} d t \int_{1}^{\lambda^{1 / a}} \frac{d s}{s} \int_{0}^{1} \sin \left(s^{a} t^{b} u^{d}\right) \frac{d u}{u} .
$$

The $u$ integral is bounded, so the integral is $O(\log \lambda)$. Now it remains to consider

$$
\begin{aligned}
V=\int_{|s| \leq 1} \frac{d s}{s} \int_{|t| \leq 1} \frac{d t}{t} \int_{|u| \leq 1} e^{i u} e^{i \lambda s^{a} t^{b} u^{d}} \frac{d u}{u} \\
=a b \int_{0}^{\lambda} \frac{d s}{s} \int_{0}^{s} \frac{d t}{t} \int_{0}^{1} \cos u \sin \left(t u^{d}\right) \frac{d u}{u} .
\end{aligned}
$$


Again the contribution from $s \leq 1$ is $O(1)$. Next

$$
\int_{0}^{1}(\cos u-1) \frac{d u}{u} \int_{1}^{\lambda} \frac{d s}{s} \int_{0}^{s} \sin \left(t u^{d}\right) \frac{d t}{t}=O(\log \lambda) .
$$

So we are down to

$$
\begin{gathered}
\int_{1}^{\lambda} \frac{d s}{s} \int_{0}^{s} \frac{d t}{t} \int_{0}^{1} \sin \left(t u^{d}\right) \frac{d u}{u}=d \int_{1}^{\lambda} \frac{d s}{s} \int_{1}^{s} \frac{d t}{t} \int_{0}^{1} \sin (t u) \frac{d u}{u} \\
=d \int_{1}^{\lambda} \frac{d s}{s} \int_{1}^{s} \frac{d t}{t} \int_{0}^{t} \sin u \frac{d u}{u}=d C \int_{1}^{\lambda} \frac{d s}{s} \int_{1}^{s} \frac{d t}{t}+O(\log \lambda)
\end{gathered}
$$

where $C=\int_{0}^{\infty} \sin u d u / u=\pi / 2$, and thus

$$
I=\frac{a b d \pi}{2}(\log \lambda)^{2}+O(\log \lambda) .
$$

\section{Edge operators}

In this section we let

$$
Q(s, t, u)=\sum_{j=1}^{L} e_{j} s^{a_{j}} t^{b_{j}} u^{d_{j}} .
$$

We assume all $e_{j}, a_{j}, b_{j}$ and $d_{j}$ are not zero, and each sequence $\left\{a_{j}\right\},\left\{b_{j}\right\}$ and $\left\{d_{j}\right\}$ is strictly monotonic. Further suppose that the $\left(a_{j}, b_{j}, d_{j}\right)$ lie on a line and that no projection of that line onto a coordinate plane passes through the origin. Finally assume $d_{1}$ and $d_{L}$ are both even at least one of $a_{1}$ and $b_{1}$ are even, and at least one of $a_{L}$ and $b_{L}$ are even. Set

$$
m_{\mathcal{R}}^{Q}(\xi)=\int_{\mathcal{R}} e^{i \xi_{4} Q(s, t, u)} \frac{d s d t d u}{s t u}
$$

where

$$
\mathcal{R}=\left\{(s, t, u):|s| \leq \frac{1}{\left|\xi_{1}\right|},|t| \leq \frac{1}{\left|\xi_{2}\right|},|u| \leq \frac{1}{\left|\xi_{3}\right|}\right\} .
$$

Let $F_{d}(s)$ be as in (1.1).

In this section we obtain the following result:

Proposition 8.1. Under the above conditions

$$
\left|m_{\mathcal{R}}^{Q}(\xi)\right| \leq C\left[\left(\sum_{i=1}^{4}|\log | \xi_{i}||\right)+1\right]
$$

and $m_{R}^{Q}$ is uniformly bounded if and only if

$$
\int_{0}^{\infty} \frac{F_{d}(s)}{s} d s=0 .
$$


Proof. Let us assume $\xi_{1}, \xi_{2}, \xi_{3}$ and $\xi_{4}$ are positive. Then $m_{\mathcal{R}}^{Q}(\xi)$ equals

$$
\int_{0}^{\frac{1}{\xi_{1}}} \int_{0}^{\frac{1}{\xi_{2}}} \int_{|u| \leq \frac{1}{\xi_{3}}}\left\{e^{i \xi_{4} Q(s, t, u)}+e^{i \xi_{4} Q(-s,-t, u)}-e^{i \xi_{4} Q(-s, t, u)}-e^{i \xi_{4} Q(s,-t, u)}\right\} \frac{d u d s d t}{u s t} .
$$

We set $u=v s^{\alpha} t^{\beta}$ with $\alpha=\frac{a_{L}-a_{1}}{d_{1}-d_{L}}$ and $\beta=\frac{b_{L}-b_{1}}{d_{1}-d_{L}}$. Then

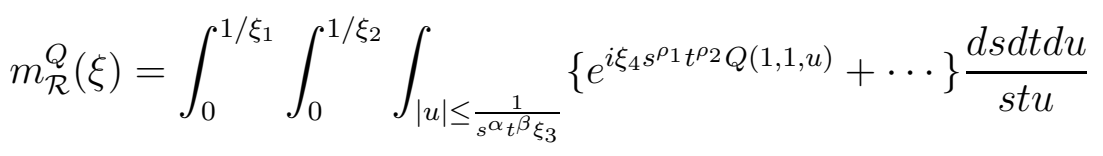

where neither $\rho_{1}$ nor $\rho_{2}$ is zero because the projection of the line through the $\left(a_{j}, b_{j}, d_{j}\right)$ onto a coordinate plane does not pass through the origin. In fact

$$
\rho_{1}=\frac{a_{L} d_{1}-a_{1} d_{L}}{d_{1}-d_{L}} \quad \text { and } \quad \rho_{2}=\frac{b_{L} d_{1}-b_{1} d_{L}}{d_{1}-d_{L}} .
$$

Let us assume first $\rho_{1}$ and $\rho_{2}$ are positive.

We now make a few more changes of variable and find $m_{\mathcal{R}}^{Q}(\xi)$ equals

$$
\int_{0}^{\frac{1}{\lambda_{1}(\xi)}} \frac{d t}{t} \int_{0}^{\frac{t}{\lambda_{2}(\xi)}} \frac{d s}{s} \int_{|u| \leq \frac{1}{s^{\circ} 1 t^{\sigma 2}}}\left\{e^{i s Q(1,1, u)}+e^{i s Q(-1,-1, u)}-e^{i s Q(-1,1, u)}-e^{i s Q(1,-1, u)}\right\} \frac{d u}{u}
$$

where $\lambda_{1}(\xi)=\xi_{2}^{\rho_{2}} \xi_{3}^{\gamma_{1}} \xi_{4}^{\gamma_{2}}, \lambda_{2}(\xi)=\xi_{1}^{\rho_{1}} \xi_{3}^{\gamma_{3}} \xi_{4}^{\gamma_{4}}, \sigma_{1}=\frac{\alpha}{\rho_{1}}$ and $\sigma_{2}=\frac{\beta}{\rho_{2}}-\frac{\alpha}{\rho_{1}}$. Once again $\sigma_{1}$ and $\sigma_{2}$ are nonzero. Let us for simplicity assume $\sigma_{1}>0$ and $\sigma_{2}>0$. The treatment for other choices of sign is similar.

We proceed in six steps:

\section{Step 1:}

$$
\int_{0}^{\lambda_{2}(\xi)}|\cdots \cdots| \frac{d t}{t}
$$

is bounded. This follows because according to Lemma 2.5, the $u$ integral is $O\left(s^{\delta}\right)$. So we may assume

$$
\lambda_{2}(\xi)<\frac{1}{\lambda_{1}(\xi)}
$$

\section{Step 2:}

$$
\int_{1}^{\infty}|\cdots \cdots| \frac{d t}{t}=O(1)
$$

From Lemma 2.5 we see the $u$ integral enjoys the estimates $O\left(s^{\delta}\right)$ and $O\left(\frac{1}{s^{\delta}}\right)$. Also $\left|e^{i s Q( \pm 1, \pm 1, u)}-1\right| \leq C\left(|u|+|u|^{\nu}\right) s$ for some $\nu>0$. So the $u$ integral has the estimate $\frac{1}{s^{\kappa} t^{\sigma_{2}}}$ for some real $\kappa$. Thus for some positive $\eta_{1}$ and positive $\eta_{2}$ the $u$ integral has the estimates $\frac{s^{\eta_{1}}}{t^{\eta_{2}}}$ and $\frac{1}{s^{\eta_{1}} t^{\eta_{2}}}$, and Step 2 follows. 


\section{Step 3:}

$$
\int_{\lambda_{2}(\xi)}^{1} \frac{d t}{t}\left|\int_{\frac{t}{\lambda_{2}(\xi)}}^{\infty} \frac{d s}{s} \int_{I}\{\cdots \cdots\} \frac{d u}{u}\right|=O(1)
$$

for any interval $I$. Step 3 follows from the fact that the $u$ integral is $O\left(\frac{1}{s^{\delta}}\right)$ (from Lemma 2.5).

Combining Steps 1, 2 and 3 we have:

Step 4:

$$
m_{\mathcal{R}}^{Q}(\xi)=\int_{\lambda_{2}(\xi)}^{\min \left(1, \frac{1}{\lambda_{1}(\xi)}\right)} \frac{d t}{t} \int_{0}^{\infty} \frac{d s}{s} \int_{|u| \leq \frac{1}{\sigma^{\sigma} t^{\sigma} 2}}\{\cdots \cdots\} \frac{d u}{u}+O(1) .
$$

Step 5:

$$
\int_{\lambda_{2}(\xi)}^{\min \left(1, \frac{1}{\lambda_{1}(\xi)}\right)} \frac{d t}{t} \int_{\frac{t}{\lambda_{2}(\xi)}}^{\infty} \frac{d s}{s} \int_{|u| \geq \frac{1}{\sigma^{\sigma} 1 t^{\sigma}}}\{\cdots \cdots\} \frac{d u}{u}=O(1) .
$$

Step 5 follows since by Lemma 2.5 the $u$ integral is $O\left(\frac{1}{s^{\delta}}\right)$.

Step 6:

$$
\int_{\lambda_{2}(\xi)}^{\min \left(1, \frac{1}{\lambda_{1}(\xi)}\right)} \frac{d t}{t} \int_{0}^{\frac{t}{\lambda_{2}(\xi)}} \frac{d s}{s} \int_{|u| \geq \frac{1}{s^{\sigma} 1 t^{\sigma}}}\{\cdots \cdots\} \frac{d u}{u}=O(1) .
$$

Again we have two estimates for the $u$ integral. First we have the estimates $s^{-\delta}$ and $s^{\delta}$ from Lemma 2.5. Secondly by van der Corput's lemma we have $s^{\kappa} t^{\sigma_{2}}$ for some real $\kappa$. Therefore we have the estimates $t^{\eta_{1}} s^{-\eta_{2}}$ and $t^{\eta_{3}} s^{\eta_{4}}$ where $\eta_{1}, \eta_{2}, \eta_{3}$, and $\eta_{4}$ are positive. This gives Step 6 .

Steps 5 and 6 show that

$$
\int_{\lambda_{2}(\xi)}^{\min \left(\frac{1}{\lambda_{1}(\xi)}, 1\right)} \frac{d t}{t} \int_{0}^{\infty} \frac{d s}{s} \int_{|u| \geq \frac{1}{s^{\sigma} 1 t^{\sigma_{2}}}}\{\cdots \cdots\} \frac{d u}{u}=O(1) .
$$

Thus

$$
m_{\mathcal{R}}^{Q}(\xi)=\int_{\lambda_{2}(\xi)}^{\min \left(1, \frac{1}{\lambda_{1}(\xi)}\right)} \frac{d t}{t} \int_{0}^{\infty} F_{d}(s) \frac{d s}{s}+O(1) .
$$

This proves Proposition 8.1 in the case that $\rho_{1}$ and $\rho_{2}$ are both positive. If $\rho_{1}$ and $\rho_{2}$ are both negative the argument is similar. In this case the $t$ integration goes from $\frac{1}{\lambda_{1}(\xi)}$ to $\infty$ and the $s$ integral goes from $\frac{t}{\lambda_{2}(\xi)}$ to $\infty$. The slight difference arises in Step 2. Now we must show

$$
\int_{0}^{1}|\cdots \cdots| \frac{d t}{t}<\infty
$$


In the case $\rho_{1}$ and $\rho_{2}$ have opposite signs, we need an extra ingredient. If $\rho_{1}<0$ and $\rho_{2}>0$, then $\sigma_{2}<0$. To see this note first that for any $i$

$$
d_{i} \sigma_{1}-1=d_{i} \frac{\alpha}{\rho_{1}}-1=\frac{d_{i} \alpha-\rho_{1}}{\rho_{1}}=-\frac{a_{i}}{\rho_{1}}>0 .
$$

Then

$$
d_{i}\left(\sigma_{1}+\sigma_{2}\right)-1=d_{i} \frac{\beta}{\rho_{2}}-1=\frac{d_{i} \beta-\rho_{2}}{\rho_{2}}=-\frac{b_{i}}{\rho_{2}}<0 .
$$

Taking differences shows $\sigma_{2}<0$. The integral to be bounded is now

$$
\int_{0}^{\frac{1}{\lambda_{1}(\xi)}} \frac{d t}{t} \int_{\lambda_{2}(\xi) t}^{\infty} \frac{d s}{s} \int_{|u| \leq \frac{1}{s^{\sigma} 1 t^{\sigma}}}\{\cdots \cdots\} \frac{d u}{u} .
$$

Now the fact that $\sigma_{2}<0$ allows us to have the estimate $s^{\gamma} t^{\eta}$ for some $\eta>0$ which we must use for small $t$. After this the argument is very similar to the argument when $\rho_{1}$ and $\rho_{2}$ are positive. If $\rho_{1}>0$ and $\rho_{2}<0$ then the extra ingredient we need is that $\sigma_{2}>0$. To see this note first

$$
d_{i} \sigma_{1}-1=d_{i} \sigma_{1}-1=-\frac{a_{1}}{\rho_{1}}<0
$$

while

$$
d_{i}\left(\sigma_{1}+\sigma_{2}\right)-1=-\frac{b_{i}}{\rho_{2}}>0
$$

and so $d_{i} \sigma_{2}>0$. Thus $\sigma_{2}>0$.

\section{Proof of sufficiency in Theorem 1.2}

In this section we complete the proof of the sufficiency part of Theorem 1.2 by establishing that the cancellation condition involving the oscillatory integral $F$ is sufficient for the boundedness of $H_{\mathcal{R}}^{P}$. We remind the reader that Theorem 1.2 requires hypotheses $(\mathrm{H} 1),(\mathrm{H} 2),(\mathrm{H} 3)$ and $(\mathrm{H} 4)$ and these assumptions will be made throughout this section. By Lemma 4.3 and Proposition 5.3, matters are reduced to showing that for each bounded edge $E$, $H_{\mathcal{R}(E)}^{Q_{E}}$ is bounded on $L^{2}\left(\mathbb{R}^{4}\right)$.

We fix a bounded edge $E$ and write $Q=Q_{E}(s, t, u)=\sum_{j=1}^{L} e_{j} s^{a_{j}} t^{b_{j}} u^{d_{j}}$ and $\mathcal{R}=\mathcal{R}(E)=\mathcal{R}_{1} \cup \mathcal{R}_{2}$ as defined in Section 5. If $\left(A_{i}, B_{i}, D_{i}\right), 1 \leq$ $i \leq m$ denote the points associated to $P$ on the 1-skeleton of the Newton polyhedron of $P$ which do not lie on $E$, then

$\mathcal{R}_{1}=\left\{|s|^{a_{1}}|t|^{b_{1}}|u|^{d_{1}} \geq|s|^{a_{2}}|t|^{b_{2}}|u|^{d_{2}},|s|^{a_{2}}|t|^{b_{2}}|u|^{d_{2}} \geq|s|^{A_{i}}|t|^{B_{i}}|u|^{D_{i}}, 1 \leq i \leq m\right\}$ 
and similarly for $\mathcal{R}_{2}$. By (H1) and (H2) we may assume that each $a_{j}, b_{j}$ and $d_{j}$ is positive and each sequence $\left\{a_{j}\right\},\left\{b_{j}\right\}$ and $\left\{d_{j}\right\}$ is strictly monotonic. Furthermore by (H3) we may suppose that the projection onto a coordinate plane of the line on which $\left(a_{j}, b_{j}, d_{j}\right)$ lie does not pass through the origin. Finally we recall that the sufficiency part of Theorem 1.2 makes an assumption on the parity of the two vertices $\left(a_{1}, b_{1}, d_{1}\right)$ and $\left(a_{L}, b_{L}, d_{L}\right)$ of the edge $E$; namely, without loss of generality, $d_{1}$ and $d_{L}$ are even, at least one of $a_{1}$ and $b_{1}$ are even and at least one of $a_{L}$ and $b_{L}$ are even.

By Proposition 6.1 it suffices to bound the oscillatory integral

$$
n_{\mathcal{R}}^{Q}=\int_{\mathcal{R}_{\xi} \cap \mathcal{R}} e^{i \xi_{4} Q(s, t, u)} \frac{d s d t d u}{s t u}
$$

uniformly in $\xi=\left(\xi_{1}, \xi_{2}, \xi_{3}, \xi_{4}\right)$. Here $\mathcal{R}_{\xi}=\left\{(s, t, u):|s| \leq 1 /\left|\xi_{1}\right|,|t| \leq\right.$ $\left.1 /\left|\xi_{2}\right|,|u| \leq 1 /\left|\xi_{3}\right|\right\}$. Without loss of generality we shall take all the $\xi_{i}$ to be positive. We decompose $n_{\mathcal{R}}^{Q}=n_{\mathcal{R}_{1}}^{Q}+n_{\mathcal{R}_{2}}^{Q}$ in the natural way. Our analysis of both $n_{\mathcal{R}_{1}}^{Q}$ and $n_{\mathcal{R}_{2}}^{Q}$ is similar to that of $m_{\mathcal{R}}^{Q}$ in Section 8. In fact we follow the same argument as for $m_{\mathcal{R}}^{Q}$ to see that

$$
n_{\mathcal{R}_{1}}^{Q}=\int_{t \in T(\xi)} \frac{d t}{t} \int_{0}^{\infty} \frac{d s}{s} \int_{u \in U} \frac{d u}{u}\left[e^{i s Q(1,1, u)}+\cdots\right]+O(1) .
$$

Here $T(\xi)=\left\{t: \lambda_{2}(\xi) \leq t \leq \min \left(\left[\lambda_{1}(\xi)\right]^{-1}, 1\right)\right\}$ where $\lambda_{1}(\xi)$ and $\lambda_{2}(\xi)$ are as in Section 8. Furthermore $U=\left\{|u| \geq 1:|u| \leq \mu_{i}(\xi) s^{M_{i}} t^{\Delta_{i}}, 1 \leq i \leq m\right\}$ where

$$
\begin{gathered}
\mu_{i}(\xi)=\xi_{4}^{-\left(E_{i}-\alpha\right)}\left[\xi_{4}^{\sigma_{1} / \sigma_{2}} \xi_{3}^{-1 / \sigma_{2}}\right]^{\Delta_{i}}, \quad M_{i}=\left(E_{i}-\alpha\right) / \rho_{1}, \\
\Delta_{i}=\frac{F_{i}-\beta}{\rho_{2}}-\frac{E_{i}-\alpha}{\rho_{1}}, \quad E_{i}=\frac{a_{2}-A_{i}}{D_{i}-d_{2}}, \quad F_{i}=\frac{b_{2}-B_{i}}{D_{i}-d_{2}}
\end{gathered}
$$

and $\alpha, \beta, \sigma_{1}, \sigma_{2}, \rho_{1}$ and $\rho_{2}$ are defined as in Section 8. We recall that (H2) guarantees that $\alpha, \beta, \sigma_{1}, \sigma_{2}, \rho_{1}, \rho_{2} \neq 0$. Furthermore (H4) implies that each $\Delta_{i} \neq 0$ (without loss of generality, we may assume $\Delta_{i}>0$ ).

Arguing as in Step 5 and Step 6 in Section 8, one finally arrives at the conclusion

$$
n_{\mathcal{R}_{1}}^{Q}=\int_{t \in \cap_{i=1}^{m-1} T_{i} \cap T_{0}} \frac{d t}{t} \int_{0}^{\infty} \frac{d s}{s} \int_{|u| \geq 1} \frac{d u}{u}\left[e^{i s Q(1,1, u)}+\cdots\right]+O(1) .
$$

Here

$$
T_{0}=\left\{t \geq 1: \xi_{1}^{\rho_{1}} \xi_{4}^{-1} \leq \xi_{4}^{\frac{E_{m}-\alpha}{\rho_{1} \Delta_{m}}} t \leq \min \left(\xi_{2}^{-\rho_{2}}, \xi_{4}^{\sigma_{1} / \sigma_{2}} \xi_{3}^{-1 / \sigma_{2}}\right)\right\}
$$


and for $1 \leq i \leq m-1$,

$$
T_{i}=\left\{t: t \geq \xi_{4}^{\frac{E_{i}-\alpha}{\rho_{1} \Delta_{i}}-\frac{E_{m}-\alpha}{\rho_{1} \Delta_{m}}}\right\} .
$$

In a similar manner one deduces that

$$
n_{\mathcal{R}_{2}}^{Q}=\int_{t \in \cap_{i=1}^{m-1} S_{i} \cap S_{0}} \frac{d t}{t} \int_{0}^{\infty} \frac{d s}{s} \int_{|u| \leq 1} \frac{d u}{u}\left[e^{i s Q(1,1, u)}+\cdots\right]+O(1)
$$

where

$$
S_{0}=\left\{t \geq 1: \xi_{1}^{\rho_{1}} \xi_{4}^{-1} \leq \xi_{4}^{\frac{G_{m}-\alpha}{\rho_{1} \Gamma_{m}}} t \leq \min \left(\xi_{2}^{-\rho_{2}}, \xi_{4}^{\sigma_{1} / \sigma_{2}} \xi_{3}^{-1 / \sigma_{2}}\right)\right\}
$$

and for $1 \leq i \leq m-1$,

$$
S_{i}=\left\{t: t \geq \xi_{4}^{\frac{G_{i}-\alpha}{\rho_{1} \Gamma_{i}}-\frac{G_{m}-\alpha}{\rho_{1} \Gamma_{m}}}\right\} .
$$

Here

$$
\Gamma_{i}=\frac{H_{i}-\beta}{\rho_{2}}-\frac{G_{i}-\alpha}{\rho_{1}}, \quad G_{i}=\frac{a_{L-1}-A_{i}}{D_{i}-d_{L-1}}, \quad H_{i}=\frac{b_{L-1}-B_{i}}{D_{i}-d_{L-2}}
$$

Therefore if we can show that for each $1 \leq i \leq m$,

$$
\frac{E_{i}-\alpha}{\rho_{1} \Delta_{i}}=\frac{G_{i}-\alpha}{\rho_{1} \Gamma_{i}}
$$

then we have

$$
n_{R}^{Q}=n_{R_{1}}^{Q}+n_{R_{2}}^{Q}=\int_{t \in \cap_{i=1}^{m-1} T_{i} \cap T_{0}} \frac{d t}{t} \int_{0}^{\infty} F(s) \frac{d s}{s}+O(1)
$$

which will conclude the proof of Theorem 1.2. To prove (9.1), it suffices to show

$$
\frac{F_{i}-\beta}{E_{i}-\alpha}=\frac{H_{i}-\beta}{G_{i}-\alpha}
$$

for each $1 \leq i \leq m$. In other words we need to see that for all $i, I_{i}+I I_{i}+$ $I I I_{i}=0$ where

$$
I_{i}=G_{i} F_{i}-H_{i} E_{i}, \quad I I_{i}=\alpha\left(H_{i}-F_{i}\right), \quad I I I_{i}=\beta\left(E_{i}-G_{i}\right) .
$$

However

$$
I_{i}+I I_{i}+I I I_{i}=\bar{L} \cdot\left(\bar{K}_{2} \times \bar{K}_{L-1}\right)
$$

where $\bar{L}=\left(a_{1}, b_{1}, d_{1}\right)-\left(a_{2}, b_{2}, d_{2}\right), \bar{K}_{2}=\left(A_{i}, B_{i}, D_{i}\right)-\left(a_{2}, b_{2}, d_{2}\right)$ and $\bar{K}_{L-1}=\left(A_{i}, B_{i}, D_{i}\right)-\left(a_{L-1}, b_{L-1}, d_{L-1}\right)$. The points $\left(a_{2}, b_{2}, d_{2}\right),\left(a_{L-1}, b_{L-1}\right.$, $\left.d_{L-1}\right)$ and $\left(A_{i}, B_{i}, D_{i}\right)$ form the vertices of a triangle and $\bar{L}$ is a multiple of $\left(a_{L-1}, b_{L-1}, d_{L-1}\right)-\left(a_{2}, b_{2}, d_{2}\right)$. Hence

$$
\bar{L} \cdot\left(\bar{K}_{2} \times \bar{K}_{L-1}\right)=0,
$$

establishing (9.1). 


\section{The necessity of the boundedness of vertex and edge operators}

If $V=\left(s^{a}, t^{b}, u^{d}\right)$ where $(a, b, d)$ is a vertex of the Newton polyhedron of $P$, we set

$$
H_{\mathbb{R}^{3}}^{V} f(x)=\iiint_{\mathbb{R}^{3}} f\left(x_{1}-s, x_{2}-t, x_{3}-u, x_{4}-s^{a} t^{b} u^{d}\right) \frac{d s d t d u}{s t u} .
$$

Let $Q=\Sigma e_{j} s^{a_{j}} t^{b_{j}} u^{d_{j}}$ where the sum is over all monomials $e_{j} s^{a_{j}} t^{b_{j}} u^{d_{j}}$ such that $\left(a_{j}, b_{j}, d_{j}\right)$ lie on a bounded edge, say $E$, of the Newton polyhedron of $P$. Set

$$
H_{\mathbb{R}^{3}}^{Q} f(x)=\iiint_{\mathbb{R}^{3}} f\left(x_{1}-s, x_{2}-t, x_{3}-u, x_{4}-Q(s, t, u)\right) \frac{d s d t d u}{s t u} .
$$

In this section $\mathcal{R}$ denotes the cube $\{(s, t, u):|s| \leq 1,|t| \leq 1|u| \leq 1\}$ in $\mathbb{R}^{3}$.

Proposition 10.1. If $H_{\mathcal{R}}^{P}$ is bounded on $L^{2}$, then $H_{\mathbb{R}^{3}}^{V}$ is bounded on $L^{2}$ for all vertices $V$, and $H_{\mathbb{R}^{3}}^{Q}$ is bounded on $L^{2}$ for all bounded edges $E$.

Remark. Lemma 7.1, Proposition 8.1 and Proposition 10.1 together show the necessity part of Theorem 1.2, and hence concludes the proof of Theorem 1.2. Furthermore, Lemma 7.1 and Proposition 10.1 show the necessity of the evenness of at least two of the exponents of each vertex in Theorem 1.1.

Proof. For $\lambda=\left(\lambda_{1}, \lambda_{2}, \lambda_{3}, \lambda_{4}\right)$ set

$$
P_{\lambda}=\lambda_{4} P\left(\frac{s}{\lambda_{1}}, \frac{t}{\lambda_{2}}, \frac{u}{\lambda_{3}}\right)
$$

and

$$
H_{\lambda} f\left(x_{1}, x_{2}, x_{3}, x_{4}\right)=\int_{\substack{|s| \leq \lambda_{1} \\|t| \leq \lambda_{2} \\|u| \leq \lambda_{3}}} f\left(x_{1}-s, x_{2}-t, x_{3}-u, x_{4}-P_{\lambda}(s, t, u)\right) \frac{d s d t u}{s t u} .
$$

If $H_{\mathcal{R}}^{P}$ is bounded, so is $H_{\lambda}$, with the same bound uniformly in $\lambda$. Also $H_{\lambda} f=D_{\lambda} * f+\bar{H}_{\lambda} f$ where $\bar{H}_{\lambda} f$ is a truncation of the triple Hilbert transform of $f$ in the first three variables and $D_{\lambda}$ is a distribution, which acting on a test function $\phi$ is given by

$$
\left\langle\phi, D_{\lambda}\right\rangle=\int_{\substack{|s| \leq \lambda_{1} \\|t| \leq \lambda_{2} \\|u| \leq \lambda_{3}}} \int_{0}^{1} \frac{\partial \phi}{\partial x_{4}}\left(s, t, u, \rho P_{\lambda}(s, t, u)\right) d \rho P_{\lambda}(s, t, u) \frac{d s d t d u}{s t u} .
$$


The latter integral is absolutely convergent since each monomial of $P$ contains positive powers of all variables.

So to prove Proposition 10.1 it suffices to find for each vertex $(a, b, d)$ a sequence of $\lambda$ 's with each component tending to infinity in such a way that

$$
\begin{aligned}
\left|P_{\lambda}(s, t, u)\right| & \leq \sum_{j} C_{j}|s|^{A_{j}}|t|^{B_{j}}|u|^{D_{j}}, A_{j}, B_{j}, D_{j}>0 \\
& \text { and } P_{\lambda}(s, t, u) \rightarrow s^{a} t^{b} u^{d} .
\end{aligned}
$$

Also for each edge we need to find a sequence of $\lambda$ 's with each component tending to infinity in such a way that

$$
\begin{aligned}
\left|P_{\lambda}(s, t, u)\right| & \leq \sum_{j} C_{j}|s|^{A_{j}}|t|^{B_{j}}|u|^{D_{j}}, A_{j}, B_{j}, D_{j}>0 \\
& \text { and } P_{\lambda}(s, t, u) \rightarrow Q(s, t, u) .
\end{aligned}
$$

The existence of this sequence of $\lambda$ 's follows from the following lemma:

Lemma 10.2. (i) Let $V=\left(s^{a}, t^{b}, u^{d}\right)$ where $(a, b, d)$ is a vertex of the Newton polyhedron of $P$. There exist positive numbers $(\alpha, \beta, \gamma)$ such that $\alpha a+\beta b+d \gamma<\alpha A+\beta B+\gamma D$ for every $(A, B, D) \neq(a, b, d)$ in the Newton polyhedron of $P$ such that $e s^{A} t^{B} u^{D}$ is a monomial of $P$.

(ii) If $Q=\Sigma e_{i} s^{a_{i}} t^{b_{i}} u^{d_{i}}$ is the polynomial corresponding to an edge of the Newton polyhedron of $P$, there exist positive $\alpha, \beta, \gamma$ such that

$$
\alpha a_{i}+\beta b_{i}+\gamma d_{i}
$$

is independent of $i$, and

$$
\alpha a_{i}+\beta b_{i}+\gamma d_{i}<\alpha A+\beta B+\gamma D
$$

for all $A, B, D$ that occur as exponents of a monomial of $P$ not lying on the edge corresponding to $Q$.

Remark. Given the lemma, we can achieve (10.1) and (10.2) by taking $\lambda_{1}=$ $\eta^{\alpha}, \lambda_{2}=\eta^{\beta}, \lambda_{3}=\eta^{\gamma}$ and $\lambda_{4}=\eta^{a \alpha} \eta^{b \beta} \eta^{\gamma d}$ for (10.1) and $\lambda_{4}=\eta^{a_{i} \alpha} \eta^{b_{i} \beta} \eta^{\gamma d_{i}}$ for (10.2). This concludes the proof of Proposition 10.1.

Proof of Lemma 10.2. To prove $(i)$, we choose a plane $\pi$ that intersects the closed Newton polyhedron only in $V$. Then since the Newton polyhedron is convex it lies entirely on one side of $\pi$. Let $N$ be the normal to $\pi$ pointing into the Newton polyhedron. Say $N=(\alpha, \beta, \gamma)$. We take this $(\alpha, \beta, \gamma)$ for the proof of $(i)$. Clearly

$$
a \alpha+\beta b+d \gamma<\alpha A+\beta B+\gamma D
$$

for every $(A, B, D) \neq(a, b, d)$ since

$$
((A-a),(B-b),(D-d)) \cdot N>0 .
$$


It remains to show that $\alpha, \beta$, and $\gamma$ are positive. To see this note that if $x-a, y-b, z-d$, are all non-negative with at least one of $x-a, y-b, z-d$ positive, $(x, y, z)$ is in the interior of the Newton polyhedron. So

$$
\alpha \cdot(x-a)+\beta \cdot(y-b)+\gamma(z-d)>0
$$

whenever $x-a \geq 0, y-b \geq 0, z-d \geq 0$ with strict inequality in one of the three cases. Thus $\alpha, \beta$ and $\gamma$ are positive.

The proof of $(i i)$ is similar. We take a plane $\pi$ which contains the line through the points $\left(a_{i}, b_{i}, d_{i}\right)$ and intersects the closed Newton polyhedron in no other points. Again we take $(\alpha, \beta, \gamma)$ so that $N=(\alpha, \beta, \gamma)$ is a normal to $\pi$ pointing into the Newton polyhedron. We just remark that we are using the assumption that the edge does not lie in a plane parallel to a coordinate plane to assert that if $(u, v, w)$ is an interior point on that edge and $x \geq u, y \geq v$, and $z \geq w$, with at least one of the inequalities strict $(x, y, z)$ is in the interior of the Newton polyhedron. This implies as above that $\alpha, \beta$ and $\gamma$ are positive.

\section{Conclusion of the proof of the necessity in Theo- rems 1.1 and 1.2}

We let, as usual,

$$
Q(s, t, u)=\sum_{j=1}^{L} e_{j} s^{a_{j}} t^{b_{j}} u^{d_{j}}
$$

with each sequence $\left\{a_{j}\right\},\left\{b_{j}\right\}$ and $\left\{d_{j}\right\}$ strictly monotone and positive. We assume at least two of $\left(a_{1}, b_{1}, d_{1}\right)$ even and at least two of $\left(a_{L}, b_{L}, d_{L}\right)$ even, say, $d_{1}$ and $d_{L}$ are even. Then with $F_{d}(s)$ as in (1.1), the following proposition will complete the proof of Theorem 1.1.

Proposition 11.1. If

$$
\int_{0}^{\infty} F_{d}(s) \frac{d s}{s}=0
$$

for all choices of $e_{j}$ with $e_{j} \neq 0$, then either all $a_{j}$ are even, all $b_{j}$ are even or all $d_{j}$ are even.

We may assume $L \geq 3$. We shall need the following lemma:

Lemma 11.2. Suppose $d_{1}$ and $d_{L}$ are even and $a_{1}$ is even. Then $a_{j}$ has the same parity as $d_{j}$ for all $j$ or all $d_{j}$ are even or all $a_{j}$ are even. Similarly is $a_{1}$ is odd, then $a_{j}$ and $d_{j}$ have opposite parity for all $j$ or all $d_{j}$ are even. 
Proof. Suppose for some $j_{0}, a_{j_{0}}$ is odd and $d_{j_{0}}$ is even. So $a_{j_{0}}-a_{1}=2 k+1$ and $d_{j_{0}}-d_{1}=2 \ell$ for some integers $k$ and $\ell$. Take a third pair $\left(a_{j_{1}}, d_{j_{1}}\right)$. Then

$$
\frac{a_{j_{0}}-a_{1}}{a_{j_{1}}-a_{1}}=\frac{d_{j_{0}}-d_{1}}{d_{j_{1}}-d_{1}}
$$

Hence $(2 k+1)\left(d_{j_{1}}-d_{1}\right)=2 \ell\left(a_{j_{1}}-a_{1}\right)$. Thus $d_{j_{1}}-d_{1}$ is even. So if for any $j_{0}, a_{j_{0}}-a_{1}$ is odd and $d_{j_{0}}-d_{1}$ is even then all $d_{j}$ are are even. This proves the lemma.

Proof of Proposition 11.1. We are assuming that $d_{1}$ and $d_{L}$ are even. Then we may assume without loss of generality that $a_{1}$ is even. Then there are two cases, $b_{1}$ is even or $b_{1}$ is odd. Assume first that $b_{1}$ is even. In this case by Lemma 11.2, we may assume in each monomial $s^{a_{j}} t^{b_{j}} u^{d_{j}}$ of $Q$ that $a_{j}, b_{j}$ and $d_{j}$ are even or $a_{j}, b_{j}$ and $d_{j}$ are odd. We write

$$
Q(s, t, u)=E(s, t, u)+H(s, t, u)
$$

where the exponents of all monomials in $E$ are even and the exponents of all monomials of $H$ are odd. We wish to show that $H$ is identically zero.

Then the condition that $\int_{0}^{\infty} F_{d}(s) \frac{d s}{s}=0$ is just

$$
\int_{0}^{\infty} \frac{d s}{s} \int_{-\infty}^{\infty} e^{i s E(1,1, u)} \sin (s H(1,1, u)) \frac{d u}{u}=0
$$

By looking at the imaginary part of the integral, we find

$$
I=\int_{0}^{\infty} \frac{d s}{s} \int_{0}^{\infty}[\sin (s E(1,1, u)) \sin (s H(1,1, u))] \frac{d u}{u}=0 .
$$

If now $b_{1}$ is odd, then in each monomial of $Q a_{j}$ and $d_{j}$ are even and $b_{j}$ is odd or $a_{j}$ and $d_{j}$ are odd and $b_{j}$ is even (unless all $d_{j}$ are even). A computation then shows that unless all $d_{j}$ are even, we again find $I=0$.

We now consider I. We interchange the order of integration and use an argument in [5], (pages 40 and 41). First note that I equals

$\int_{0}^{\infty} \frac{d u}{u} \int_{0}^{\infty} \frac{d s}{s}\{\cos (s[(E(1,1, u)+H(1,1, u))])-\cos (s[(E(1,1, u)-H(1,1, u))])\}$.

Let

$$
A=|E(1,1, u)+H(1,1, u)|^{-1} \quad \text { and } \quad B=|E(1,1, u)-H(1,1, u)|^{-1} .
$$


Then we write

$$
\begin{aligned}
\int_{\epsilon}^{\infty} \frac{d s}{s}\{\cos (s[E(1,1, u) & +H(1,1, u)])\}= \\
= & \int_{\epsilon}^{A}\{\cos (s[E(1,1, u)+H(1,1, u)])-1\} \frac{d s}{s} \\
& +\int_{A}^{\infty}\{\cos (s[E(1,1, u)+H(1,1, u)])\} \frac{d s}{s}+\int_{\epsilon}^{A} \frac{d s}{s}
\end{aligned}
$$

and

$$
\begin{aligned}
\int_{\epsilon}^{\infty} \frac{d s}{s}\{\cos (s[E(1,1, u) & -H(1,1, u)])\}= \\
= & \int_{\epsilon}^{B}\{\cos (s[E(1,1, u)-H(1,1, u)])-1\} \frac{d s}{s} \\
& +\int_{B}^{\infty}\{\cos (s[E(1,1, u)-H(1,1, u)])\} \frac{d s}{s}+\int_{\epsilon}^{B} \frac{d s}{s} .
\end{aligned}
$$

So we have

$$
I=\int_{0}^{\infty} \frac{d u}{u} \log \left|\frac{E(1,1, u)+H(1,1, u)}{E(1,1, u)-H(1,1, u)}\right| .
$$

But if we take all $e_{j}$ positive then

$$
|E(1,1, u)+H(1,1, u)| \geq|E(1,1, u)-H(1,1, u)|
$$

and at least for some interval of $u$

$$
|E(1,1, u)+H(1,1, u)|>|E(1,1, u)-H(1,1, u)|
$$

unless $H$ is identically zero. If $H$ is not identically zero, then since $I=0$ we arrive at a contradiction. This completes the proof of Proposition 11.1 and also of Theorem 1.1.

\section{Two Examples}

In this section we consider two examples of edge operators. The polynomials are

$$
Q_{1}=A s^{30} t^{29} u^{2}+C s^{27} t^{28} u^{3}+D s^{21} t^{26} u^{5}+B s^{18} t^{25} u^{6}
$$

and

$$
Q_{2}=A s^{2} t^{2} u^{4}+B s^{3} t^{5} u^{3}+D s^{4} t^{8} u^{2} .
$$


We consider

$$
H_{1} f(x)=\iiint_{\mathbb{R}^{3}} f\left(x_{1}-s, x_{2}-t, x_{3}-u, x_{4}-Q_{1}(s, t, u)\right) \frac{d s d t d u}{s t u},
$$

and

$$
H_{2} f(x)=\iiint_{\mathbb{R}^{3}} f\left(x_{1}-s, x_{2}-t, x_{3}-u, x_{4}-Q_{2}(s, t, u)\right) \frac{d s d t d u}{s t u} .
$$

We shall show that for $H_{1}$, there is a choice of $(A, C, D, B)$ for which none of $A, C, D, B$ are zero and $H_{1}$ is bounded on $L^{2}\left(\mathbb{R}^{4}\right)$ while if $A>0, C>0$, $D>0$, and $B>0, H_{1}$ is not bounded on $L^{2}$. On the other hand for every choice of $A, B, D$ with $A \neq 0, B \neq 0$, and $D \neq 0, H_{2}$ is unbounded on $L^{2}$.

By Proposition 8.1 in each case it suffices to see whether $\int_{0}^{\infty} F_{d}(s) d s / s=0$. Moreover, in Section 11, we showed that if all of the coefficients are positive then $\int_{0}^{\infty} F_{d}(s) d s / s \neq 0$.

We shall first show that if $A=B$ and $C=-D$ in $Q_{1}$, then $\int_{0}^{\infty} F_{d}(s) d s / s$ $=0$. With the notation of Section 11,

$$
E(u)=A u^{2}+B u^{6} \text { and } H(u)=C u^{3}+D u^{5},
$$

and

$$
\begin{aligned}
\int_{0}^{\infty} \frac{F(s)}{s} d s & =\int_{0}^{\infty} \frac{d s}{s} \int_{0}^{\infty} \sin (s E(u)) \sin (s H(u)) \frac{d u}{u} \\
& =\int_{0}^{\infty} \log \frac{|E(u)+H(u)|}{|E(u)-H(u)|} \frac{d u}{u} \\
& =\int_{0}^{1} \log \frac{|E(u)+H(u)|}{|E(u)-H(u)|} \frac{d u}{u}+\int_{0}^{1} \log \frac{\left|E\left(\frac{1}{u}\right)+H\left(\frac{1}{u}\right)\right|}{\left|E\left(\frac{1}{u}\right)-H\left(\frac{1}{u}\right)\right|} \frac{d u}{u} \\
& :=I+I I .
\end{aligned}
$$

Now

$$
I I=\int_{0}^{1} \log \frac{\left|u^{8}\left(E\left(\frac{1}{u}\right)+H\left(\frac{1}{u}\right)\right)\right|}{\left|u^{8}\left(E\left(\frac{1}{u}\right)-H\left(\frac{1}{u}\right)\right)\right|} \frac{d u}{u} .
$$

Since $A=B$ and $C=-D$, we see that

$$
I I=\int_{0}^{1} \log \frac{|E(u)-H(u)|}{|E(u)+H(u)|} \frac{d u}{u}=-I .
$$

This completes the analysis of $H_{1}$. 
For $H_{2}$,

$$
\int_{0}^{\infty} \frac{F(s)}{s} d s=\int_{0}^{\infty} \frac{d s}{s} \int_{0}^{\infty} e^{i\left(A u^{4}+D u^{2}\right) s} \sin \left(B u^{3} s\right) \frac{d u}{u} .
$$

After a change of variables one sees that for some non-zero $\lambda$ depending on $A, B$ and $D$,

$$
\int_{0}^{\infty} \frac{F(s)}{s} d s=\int_{0}^{\infty} \frac{\sin \lambda s}{s} \int_{0}^{\infty} e^{i\left(u+\frac{1}{u}\right) s} \frac{d u}{u} d s
$$

or

$$
\int_{0}^{\infty} \frac{F(s)}{s} d s=\int_{0}^{\infty} \frac{\sin \lambda s}{s} \int_{0}^{\infty} e^{i\left(u-\frac{1}{u}\right) s} \frac{d u}{u} d s
$$

depending on the signs of $A$ and $D$. The imaginary part of the first integral reduces to

$$
\int_{0}^{\infty} \frac{d s}{s} \sin (\lambda s) \int_{0}^{1} \sin \left(\left(\frac{1}{u}+u\right) s\right) \frac{d u}{u}=\int_{0}^{1} \log \left|\frac{\lambda-\left(u+\frac{1}{u}\right)}{\lambda+\left(u+\frac{1}{u}\right)}\right| \frac{d u}{u}
$$

which is non-zero for any $\lambda \neq 0$.

The second integral reduces to

$$
\begin{aligned}
\int_{0}^{\infty} \frac{d s}{s} \sin \lambda s \int_{0}^{1} \cos \left(\left(u-\frac{1}{u}\right) s\right) \frac{d u}{u} & = \\
& =\int_{0}^{1}\left\{\operatorname{sgn}\left(\left(\frac{1}{u}-u\right)+\lambda\right)+\operatorname{sgn}\left(\lambda-\left(\frac{1}{u}-u\right)\right)\right\} \frac{d u}{u}
\end{aligned}
$$

which is non-zero for any $\lambda \neq 0$.

\section{References}

[1] Carbery, A., Wainger, S. And Wright, J.: Double Hilbert transforms along polynomial surfaces in $\mathbb{R}^{3}$. Duke Math. J. 101 (2000), no. 3, 499-513.

[2] Nagel, A. And Wainger, S.: $L^{2}$ boundedness of Hilbert transform along surfaces and convolution operators homogeneous with respect to a multi parameter group. Amer. J. Math. 99 (1977), no. 4, 761-785.

[3] Patel, S. K.: Double Hilbert transforms. PhD thesis, University of Edinburgh, 2004.

[4] Ricci, F. And Stein, E. M.: Multiparameter singular integrals and maximal functions. Ann. Inst. Fourier (Grenoble) 42 (1992), no. 3, 637-670.

[5] Stein, E. M.: Singular integrals and differentiability properties of functions. Princeton Mathematical Series 30. Princeton University Press, Princeton, N.J., 1970. 
[6] Stein, E. M. And Wainger, S.: The estimation of an integral arising in multiplier transformations. Studia Math. 35 (1970), 101-104.

[7] Stein, E. M. and Wainger, S.: Problems in harmonic analysis related to curvature. Bull. Amer. Math. Soc. 84 (1978), no. 6, 1239-1295.

Recibido: 27 de junio de 2007

Anthony Carbery

School of Mathematics and Maxwell Institute for Mathematical Sciences

University of Edinburgh Edinburgh EH9 3JZ, Scotland

A.Carbery@ed.ac.uk

Stephen Wainger

Department of Mathematics

University of Wisconsin at Madison

Madison WI 53706, U.S.A.

wainger@math . wisc .edu

James Wright

School of Mathematics and Maxwell Institute for Mathematical Sciences University of Edinburgh Edinburgh EH9 3JZ, Scotland

J.R.Wright@ed.ac.uk

The second author was supported in part by NSF grant DMS-0555850 at the University of Wisconsin-Madison. The third author was supported in part by an EPSRC grant. 